\title{
Global distributions of overlapping gravity waves in HIRDLS data
}

\author{
C. J. Wright ${ }^{1,2}$, S. M. Osprey ${ }^{3}$, and J. C. Gille ${ }^{2,4}$ \\ ${ }^{1}$ Centre for Space, Atmosphere and Ocean Science, University of Bath, Claverton Down, Bath, UK \\ ${ }^{2}$ Atmospheric Chemistry Division, National Center for Atmospheric Research, Boulder, CO, USA \\ ${ }^{3}$ Atmospheric, Oceanic and Planetary Physics, Department of Physics, University of Oxford, Oxford, UK \\ ${ }^{4}$ Center for Limb Atmospheric Sounding, University of Colorado, Boulder, CO, USA
}

Correspondence to: C. J. Wright (corwin.wright@trinity.oxon.org)

Received: 17 October 2014 - Published in Atmos. Chem. Phys. Discuss.: 17 February 2015

Revised: 29 May 2015 - Accepted: 6 July 2015 - Published: 30 July 2015

\begin{abstract}
Data from the High Resolution Dynamics Limb Sounder (HIRDLS) instrument on NASA's Aura satellite are used to investigate the relative numerical variability of observed gravity wave packets as a function of both horizontal and vertical wavenumber, with support from the Sounding of the Atmosphere using Broadband Emission Radiometry (SABER) instrument on TIMED. We see that these distributions are dominated by large vertical and small horizontal wavenumbers, and have a similar spectral form at all heights and latitudes, albeit with important differences. By dividing our observed wavenumber distribution into particular subspecies of waves, we demonstrate that these distributions exhibit significant temporal and spatial variability, and that small-scale variability associated with particular geophysical phenomena such as the monsoon arises due to variations in specific parts of the observed spectrum. We further show that the well-known Andes/Antarctic Peninsula gravity wave hotspot during southern winter, home to some of the largest wave fluxes on the planet, is made up of relatively few waves, but with a significantly increased flux per wave due to their spectral characteristics. These results have implications for the modelling of gravity wave phenomena.
\end{abstract}

\section{Introduction}

Gravity waves (GWs) are a key component in our understanding of the global atmospheric circulation, helping to determine the broad-scale structure of the middle atmosphere and driving atmospheric dynamics on all scales (e.g. Fritts, 1984; Holton et al., 1995; Nappo, 2002; Fritts and Alexander, 2003 , and references therein). Vertically propagating GWs carry a vertical flux of horizontal pseudomomentum (momentum flux, MF), transferring it away from low altitudes and returning it to the mean flow at altitudes and locations far removed from the region of wave generation. Parameterisations of these processes used for numerical weather prediction and climate models have significantly reduced circulation biases (e.g. Karoly et al., 1996; Alexander et al., 2010; Geller et al., 2013).

The propagation characteristics of GWs, such as their phase velocity and their direction, are difficult to directly measure from current almost-instantaneous satellite data. Nevertheless, these waves can often be effectively parameterised using spatial information, such as their horizontal and vertical wavenumbers $k_{h}=1 / \lambda_{h}$ and $k_{z}=1 / \lambda_{z}{ }^{1}$. Key processes such as Doppler shifting, critical-level wave filtering, and ducting act to redistribute wave energy and momentum in ways which are dependent on these spectral characteristics. Accordingly, a better knowledge of the wavenumber distribution of these signals in the real atmosphere could aid significantly in our understanding of the atmospheric system, providing necessary observational constraints for the GW parameterisations which form a key component of climate and weather models (e.g. Kim et al., 2003; Song et al., 2007; Richter et al., 2010) and, perhaps more directly, in diagnosing the performance of current and future high-resolution

\footnotetext{
${ }^{1}$ We use this definition of wavenumber, with units of wave cycles per metre (cpm), throughout this study. This is in order to allow simple conversion between wavelength and wavenumber. Many other studies instead define wavenumber as this value multiplied by a factor of $2 \pi$, giving units of radians per metre; this is dimensionally equivalent.
} 
models which attempt to simulate waves at the scales accessible to modern satellite instrumentation.

Recent advances in satellite instrumentation (e.g. Fritts and Alexander, 2003; Wu et al., 2006; Preusse et al., 2008; Alexander et al., 2010, and references therein) have made possible the direct detection and measurement of GWs on a global scale at resolutions previously unavailable, allowing for identification of their geographic distribution and their spectral characterisation. In this article, we use measurements from the High Resolution Dynamics Limb Sounder (HIRDLS) on NASA's Aura satellite to produce a broadscale assessment of the wavenumber distribution in the stratosphere and lower mesosphere throughout the calendar year 2007. Specifically, we use data derived using the Stockwell transform (ST, Stockwell et al., 1996) as applied to atmospheric temperature profiles; this technique has been used extensively for the detection of gravity waves in atmospheric data (e.g. Stockwell, 1999; Wang et al., 2006; Alexander et al., 2008; Wright, 2012; McDonald, 2012; France et al., 2012). Finally, we use these data to examine the variations between four "species" of gravity waves defined by their range of horizontal and vertical wavenumbers and analyse these independently, showing both similarities and differences in their temporal and spatial behaviour.

Section 2 discusses the instruments used, Sect. 3 describes the analysis method, and Sect. 4 gives a brief summary of the most important limitations that apply to our results. Sections 5 and 6 then discuss the observed one-dimensional and two-dimensional wave spectra in the global mean respectively, and Sect. 7 regional variations. Finally, Sect. 8 divides the observed wave population into species and discusses their variation.

\section{Data}

\subsection{HIRDLS}

Designed to measure high vertical resolution atmospheric radiance profiles, HIRDLS (Gille et al., 2003) is a 21-channel limb-scanning filter radiometer on NASA's Aura satellite.

Shortly after launch in 2004 an optical blockage, believed to be a loosened flap of the Kapton ${ }^{\circledR}$ material lining the foreoptics section of the instrument, was found to obscure around $80 \%$ of the viewing aperture. Consequently, major corrective work has been required to produce useful atmospheric data. Measurements of temperature, cloud, and a wide range of chemical species have now been successfully retrieved and made available for scientific analysis (Massie et al., 2007; Nardi et al., 2008; Kinnison et al., 2008; Gille et al., 2008, 2013; Khosravi et al., 2009).

One particularly productive area of research has been the detection and analysis of GWs (e.g. Alexander et al., 2008; Hoffmann and Alexander, 2009; Wang and Alexander, 2009; Wright et al., 2010, 2013; Yan et al., 2010; France et al.,
2012; Ern and Preusse, 2012). This would have been possible with the original horizontal and vertical scanning mode of the instrument, but the closer along-track profile spacing made possible by the lack of horizontal scanning capability has allowed measurements to be taken at a higher horizontal resolution than originally planned, facilitating such research. Measurements are made in vertical profiles: around 5500 profiles are obtained per day, spaced approximately 70 $105 \mathrm{~km}$ apart depending on the scan direction (see Sect. 4.3) and scanning pattern used. Due to the optical blockage, measurements are taken at a significant horizontal angle, $\sim 47^{\circ}$, to the track of the satellite, as a result of which observations cannot be made south of $62^{\circ} \mathrm{S}$ and are not spatially co-located with other instruments on the Aura satellite.

V007 of the HIRDLS data set provides vertical temperature profiles from the tropopause to $\sim 80 \mathrm{~km}$ in altitude as a function of pressure, allowing us to produce useful gravity wave analyses at these higher altitudes. Measurements have a precision $\sim 0.5 \mathrm{~K}$ throughout the lower stratosphere, increasing with height to $\sim 1 \mathrm{~K}$ at the stratopause and $3 \mathrm{~K}$ or more above this, depending on latitude and season (Gille et al., 2013). Vertical resolution is $\sim 1 \mathrm{~km}$ throughout the stratosphere, smoothly declining to $\sim 2 \mathrm{~km}$ above this.

Data are available from late January 2005 until March 2008, when a failure of the optical chopper terminated data collection. A variety of scanning modes were used until June 2006, after which the scanning mode remained constant until the end of the mission. Consequently, we examine here data from the calendar year 2007; this provides a complete year of data at a consistent horizontal resolution, but without biasing the results by including an additional fractional year.

\subsection{SABER}

In parts of this paper, we also use data derived from the Sounding of the Atmosphere using Broadband Emission Radiometry (SABER) instrument on NASA's TIMED satellite to assess the methodology. A 10-channel limb-sounding infrared radiometer, SABER, was intended primarily to measure and characterise the mesosphere and lower thermosphere on a global scale, but also scans down into the stratosphere, providing $\sim 2200$ vertical profiles per day with a vertical resolution of approximately $2 \mathrm{~km}$ (Mertens et al., 2009) and an along-track profile spacing alternating between $\sim 200$ and $\sim 550 \mathrm{~km}$ depending on scan direction (in an equivalent manner to HIRDLS, discussed in Sect. 4.3 - see Fig. 1 of Ern et al., 2011, for a diagram illustrating the comparative scanning pattern of both instruments). Kinetic temperature profiles cover the altitude range from $\sim 15$ to $\sim 120 \mathrm{~km}$ (Mertens et al., 2009; Wrasse et al., 2008).

SABER's scanning routine incorporates the TIMED spacecraft's yaw cycle, with the coverage region shifting north and south every 60 days to cover the poles alternately. Accordingly, while the coverage of the instrument in the tropics and at midlatitudes remains constant throughout the 
year, high northerly and southerly latitudes are only covered for 60 of every 120 days, in a 60-day on, 60-day off cycle, with coverage in the "off" hemisphere extending to $54^{\circ}$ and in the "on" hemisphere to $87^{\circ}$. We use SABER version 1.07 temperature data for 2002-2012, with a precision of $\sim 0.8 \mathrm{~K}$ (Remsberg et al., 2008); the longer period is possible due to the consistent profile-to-profile scanning pattern of the instrument since launch, but provides a smaller total number of resolved wavelike features due to the coarser resolution of the data set.

\section{Analysis}

To detect gravity waves, we use the method of Alexander et al. (2008), as modified by Wright and Gille (2013). Briefly, we compute the daily mean background temperature and first seven planetary-scale wave modes at each height level using a Fourier transform in longitude separately for each $2^{\circ}$ latitude band, and remove these from the data (Fetzer and Gille, 1994). This leaves temperature perturbation profiles from the surface to $80 \mathrm{~km}$. Below clouds, the temperature data set relaxes back to the GEOS-5 a priori data, and consequently we do not expect to detect meaningful gravity wave signals at these levels, but we include this information to provide some overage for the analysis; this may suppress detection of the longest vertical-wavelength waves in our analysis at tropical latitudes to some degree at the $20 \mathrm{~km}$ altitude level, and may lead to a slight low-biasing of wave amplitude at the lower altitudes of our analysis, but should not otherwise impact upon our results. We further add 20 vertical levels of zero-padding at each end of the vertical domain to reduce wraparound and Gibbs-ringing effects. We then interpolate onto a regular $1 \mathrm{~km}$ vertical scale, representative of the resolution of the instrument at most altitudes, and transform the profile using the Stockwell transform (ST, Stockwell, 1999). This returns, for each height and wavenumber considered, a phase and wave amplitude for any wavelike signals detected. For further discussion of this, see e.g. Sect. 2.2 of Alexander et al. (2008).

We next cross-multiply along-track adjacent profile pairs to compute complex co-spectra, from which we compute the co-varying temperature amplitude $\hat{T}\left(k_{z}, z\right)$, and locate each $\hat{T}\left(k_{z}, z\right)$ at which a distinct local maximum is observed in the ST spectrum. We then apply the statistical significance test described by McDonald (2012), modified as described by Wright and Gille (2013), and require signals to be significant at the $99 \%$ level. From this analysis we obtain, for each height level in each profile, an estimate for each statistically significant wavelike signal (hereafter simply "wave") for the parameters $\hat{T}$ and $k_{z}$. Since multiple above-noise spectral peaks may exist in a profile at a given height, this method allows for the detection of overlapping wavelike signals, in contrast to many previous studies. For each of these signals, using the phase change between these signals $\Delta \phi_{i, i+1}$ and profile separation $\Delta r_{i, i+1}$, we further compute and retain the horizontal wavenumber

$k_{h}=\frac{1}{2 \pi} \frac{\Delta \phi_{i, i+1}}{\Delta r_{i, i+1}}($ cycles per metre, $\mathrm{cpm})$.

Values of $k_{z}$ returned from the analysis are quantised; these values are "binned" into bins corresponding to each quantised value, and all bins are shown on all relevant figures. Values of $k_{h}$ form a continuous spectrum and are binned in all analyses into 50 bins base-10-logarithmically distributed across the range $10^{-7}-10^{-5} \mathrm{~m}^{-1}$. Changing the number of bins in $k_{h}$ does not significantly alter the form of the distribution; the value of 50 bins was chosen to provide a balance between data resolution and local data processing capabilities.

Finally, in some sections, we use momentum flux estimated from these measurements. Momentum flux is important in both the real and model worlds, both as a real-world mechanism which teleconnects sections of the atmosphere without mass transfer and in the model world as a property which when parameterised at the subgrid level helps to correct for momentum and energy biases arising due to the lack of simulation of small-scale waves and related processes. This is calculated as (Ern et al., 2004)

$M_{i, i+1}=\frac{\rho}{2} \frac{k_{h}}{k_{z}}\left(\frac{g}{N}\right)^{2}\left(\frac{\hat{T}}{\bar{T}}\right)^{2}$,

where $\rho$ is the local atmospheric density, $g$ is the acceleration due to gravity, $N$ is the buoyancy frequency, and $\bar{T}$ is the local mean atmospheric temperature. It should be noted that this expression only applies well under the midfrequency approximation (i.e. the assumption that $N \gg \hat{\omega} \gg f$ ) for gravity waves, and does not take account of vertical shear or of reflection (e.g. Sato and Dunkerton, 1997). The midfrequency approximation applies well to our data in most spectral regions, except for those at very high vertical and short horizontal wavenumbers.

It should be noted that many, if not the majority, of these signals are likely to be observations of the same real wave structure at adjacent height levels and, in cases where the real wave is aligned in a similar direction to the satellite scan track, in adjacent profiles. This distinction is very important and should be carefully considered when summing measured signals, especially from long horizontal waves. Accordingly, in sections where we would sum measured signals in the vertical, we take a single height level rather than a range, to avoid introducing this bias into our results. This is harder to compensate for in the horizontal since it would require full identification of distinct multi-profile wave packets, and is not attempted here; this should accordingly be taken as a caveat to our horizontal wavelength results, which will exhibit some bias towards longer wavelengths. Although this can cause issues with counting wave packets as we do here, this is not entirely a negative feature of the data set overall 

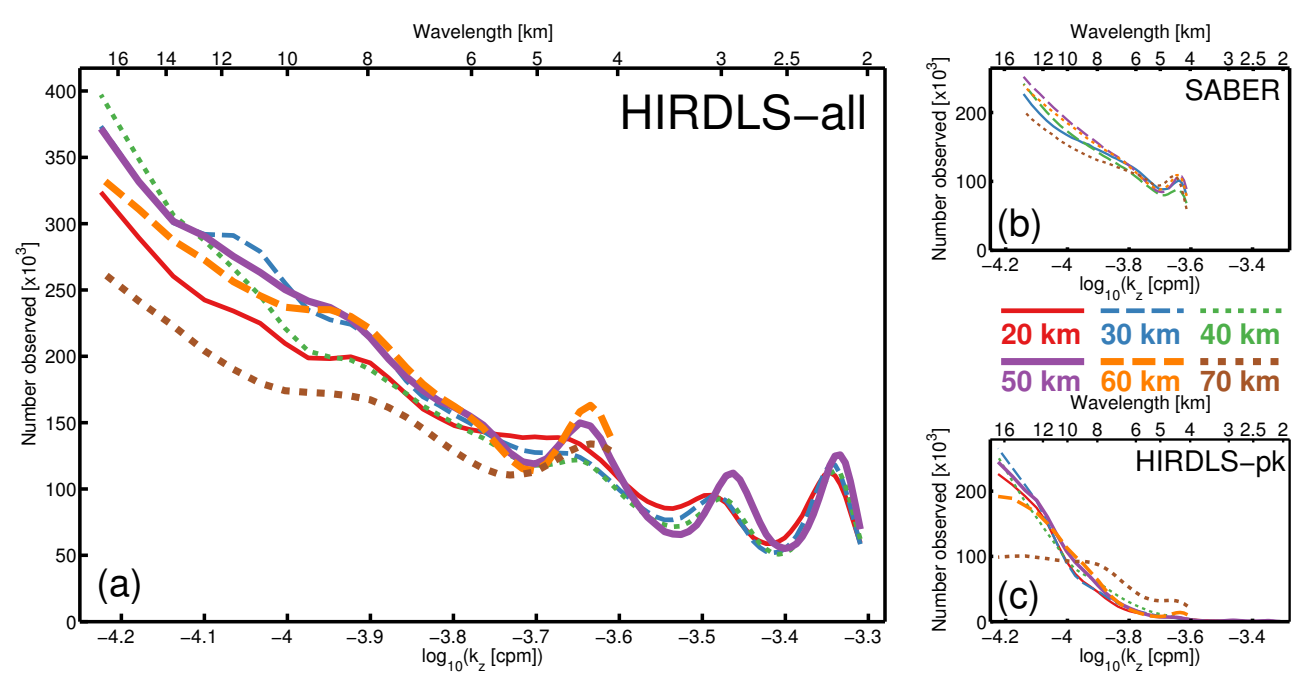

Figure 1. (a) Number of observed waves (all times, all locations), as a function of the $\log _{10}$ vertical wavenumber. Solid coloured lines indicate the number of observed waves at each wavenumber for six selected heights (key at centre right). (b) Equivalent results for SABER. Note that the $20 \mathrm{~km}$ line is omitted here due to poor data quality at this altitude. (c) Equivalent result for the HIRDLS-pk data set.

when used for other purposes; features of a large physical scale such that they would be detected in multiple profiles will inherently contribute more to area averages of physical parameters than features of a smaller physical scale, and this inherent repeated sampling to some degree therefore weights these features more strongly than smaller ones.

\subsection{HIRDLS-pk}

In Figs. 1 and 3, we compare our results to a data set computed using the same HIRDLS V007 data, but locating only the single largest-co-varying-amplitude peak at each altitude level of each profile. This reduces our data set to one almost identical to Alexander et al. (2008), with minor differences due to (i) applying the noise-comparison method of McDonald (2012), (ii) the use of a Fourier transform rather than a Stockwell transform to remove planetary-scale waves, and (iii) the data extending up to $80 \mathrm{~km}$ altitude. We refer to this data set as "HIRDLS-pk" and to the primary data set as "HIRDLS-all" where necessary to avoid ambiguity.

\subsection{SABER}

Figures 1 and 3 also compare our data to equivalent results computed using SABER. This analysis is methodologically equivalent to the main HIRDLS analysis method, with three differences: (i) due to the smaller numbers of usable profiles, we compute planetary waves based on a rolling 3-day mean of global temperature rather than individual days, (ii) data are interpolated to a $2 \mathrm{~km}$ vertical resolution rather than $1 \mathrm{~km}$, and (iii) after Ern et al. (2011), we remove profile pairs separated by more than $300 \mathrm{~km}$. We refer to this data set as "SABER".

\section{Measurement limitations}

Our measured results will not fully capture the true spectrum of wavelength data, due to the observational filter (Alexander, 1998; Preusse et al., 2000, 2008; Trinh et al., 2015) of the instrument and to other effects arising as a result of the analysis process. We discuss here the key limitations inherent in our measurements and analysis technique; Table 1 summarises these effects.

We describe here only the limitations applying to HIRDLS; a full description of the applicable limitations for SABER is omitted for brevity. Generally, such limitations are similar after allowing for the different instrument resolutions and weighting functions, and identical for the effects discussed in Sect. 4.4-4.7, with the exception of the specific boundary latitude values given in Sect. 4.4

\subsection{Vertical resolution}

For HIRDLS, the lower limit to measurable vertical wavelength $\lambda_{z}$ in the stratosphere is $\sim 2 \mathrm{~km}$ (e.g. Wright et al., 2011). This is imposed by the $1 \mathrm{~km}$ vertical resolution of the data, resulting from the radiometer channel detectors, low radiometric noise and the choice of vertical sampling (Gille et al., 2008), which result in narrow averaging kernels (Khosravi et al., 2009). This corresponds to an upper limit on vertical wavenumber $k_{z}=5 \times 10^{-4}$ cycles per metre (cpm). Due to the increased vertical width of the averaging kernels at higher altitudes (Gille et al., 2013), this increases to $\lambda_{z} \sim 3.5 \mathrm{~km}$ (decreases to $2.9 \times 10^{-4} \mathrm{cpm}$ ) at mesospheric altitudes. We impose a lower limit of $k_{z}=6.25 \times 10^{-5} \mathrm{cpm}$ (upper limit of $\lambda_{z}=16 \mathrm{~km}$ ) in our analysis; the underlying reasons for this relate to particular features of our results, and are consequently discussed in Sect. 5.1. 
Table 1. Summary of errors in measurements due to analysis method. F indicates a fixed limit, U an uncertainty. * indicates that the asterisked number approximately doubles above $60 \mathrm{~km}$; $\$$ indicates that this value is due to choices we have made rather than physical or methodological limitations.

\begin{tabular}{lcll}
\hline Vertical wavelength & & & \\
\hline Instrument resolution & $\mathrm{F}$ & Lower bound & $2 * \mathrm{~km}$ \\
Analysis option & $\mathrm{F} \$$ & Lower bound & $16 \mathrm{~km}$ \\
S-Transform analysis & $\mathrm{U}$ & Fully resolved waves & $\pm<10 \%$ \\
& $\mathrm{U}$ & $>1 / 2$ resolved & $-<20 \%$ \\
& $\mathrm{U}$ & $<1 / 2$ resolved & $->20 \%$ \\
Propagation direction & $\mathrm{U}$ & Unknown & Measurement is upper bound to true value \\
Aliasing & $\mathrm{U}$ & Unknown & Shifts waves below resolvable $\lambda_{z}$ to larger values \\
\hline Horizontal wavelengths & & & \\
\hline Profile separation & $\mathrm{F}$ & Lower bound & $140-215 \mathrm{~km}$, varies with scan direction and $z$ \\
Weighting functions & $\mathrm{F}$ & Lower bound & $20-200 \mathrm{~km}$, depending on propagation angle \\
Planetary wave removal & $\mathrm{F} \$$ & Upper bound & $5700 \mathrm{~km}$ (mode-7) zonal at Equator, varies with $\phi$ \\
Analysis option & $\mathrm{F} \$$ & Upper bound & $10000 \mathrm{~km}$ along-track \\
$\Delta \phi$ measurement & $\mathrm{U}$ & $2^{*}<\lambda_{z}<4^{*} \mathrm{~km}$ & $\pm<25 \%$ \\
& $\mathrm{U}$ & $\lambda_{z}>4^{*} \mathrm{~km}$ & $\pm<10 \%$ \\
Propagation direction & $\mathrm{U}$ & Unknown & Measurement is upper bound to true value \\
Aliasing & $\mathrm{U}$ & Unknown & Shifts waves below resolvable $\lambda_{h}$ to larger values \\
\hline Temperature perturbations & & & \\
\hline Amplitude sensitivity & $\mathrm{U}$ & $\lambda_{h}>300, \lambda_{z}<4 \mathrm{~km}$ & $-<60 \%$ \\
& $\mathrm{U}$ & Otherwise & $->60 \%$ \\
S-Transform analysis & $\mathrm{U}$ & Fully resolved waves & $-<25 \%$ \\
& $\mathrm{U}$ & Not fully resolved & $->25 \%$ \\
\hline
\end{tabular}

\subsection{Horizontal resolution}

The 70-105 km separation between profiles in principle imposes a maximum resolvable $k_{h}$ of $4.8-7.1 \times 10^{-6} \mathrm{cpm}$ (minimum resolvable $\lambda_{h}$ of 140-215 km) depending on the scanning pattern used (see Sect. 4.3 for further details). However, limb-sensing techniques have very broad horizontal weighting functions, which imply a significant horizontal averaging. For HIRDLS, this is around $200 \mathrm{~km}$ in the line-of-sight (LOS) direction and $10 \mathrm{~km}$ in the direction perpendicular to this. Hence, whilst the instrument is in principle capable of detecting short waves propagating in a horizontal direction perpendicular to the LOS, waves propagating along the LOS shorter than $\sim 200 \mathrm{~km}$ will not be detected regardless of the actual profile spacing for these profiles (Alexander et al., 2008). Adjacent profile weighting functions do not overlap.

In practice, waves with large $k_{h}$ will be much more challenging to detect. Preusse et al. (2000) and Sect. 2 of Preusse et al. (2002) discuss this for the CRISTA instrument, with broad applicability for all limb-sounding instruments including HIRDLS, and predict that a drop in sensitivity at large $k_{h}$ will be observed. This decline in measured amplitude is strongly related to the $k_{z}$ of the signal in question: at the largest $k_{h}$, waves with smaller $k_{z}$ will tend to be detected with somewhat smaller amplitudes than an otherwise identical wave with larger $k_{z}$.
The minimum resolvable horizontal wavenumber is somewhat harder to determine theoretically. For waves aligned perfectly in the zonal direction, where we filter out signals based on planetary waves of mode 7 or below, the minimum $k_{h}$ will correspond to that of a mode-7 planetary wave, i.e. $1.7 \times 10^{-7} \mathrm{cpm}$ at the Equator and higher at higher latitudes. However, in practice, the satellite scan track will be aligned in the zonal direction only at the poles and in a meridional or near-meridional direction for most of its orbit, and wavelengths longer than this will not be filtered out in these directions. We impose a cutoff of $k_{h}=1 \times 10^{-7} \mathrm{cpm}$ $\left(\lambda_{h}=10000 \mathrm{~km}\right)$ on our analysis; however, such a wave would imply $\Delta \phi_{i, i+1} \sim(2 \pi / 100)$ radians, which is likely to be well below any practically resolvable phase difference between two profiles, and accordingly very small horizontal wavenumbers in our results should be treated with extreme caution both because of this and because they are likely to have a large relative angle of propagation (see Sect. 4.4 below), producing a measured value much smaller than the true horizontal wavenumber of the wave.

Some variation in the maximum resolvable $k_{h}$ exists due to variations in the instrument scan pattern over the mission. To avoid this we use only data from 2007, when the scanning pattern remained consistent: specifically, from June 2006 onwards, HIRDLS obtained 27 pairs of vertical up and down 
scans of $\sim 31 \mathrm{~s}$ duration each, followed by a $1-2 \mathrm{~s}$ space view before the next 27 scan pairs (Gille et al., 2013).

\subsection{Scan duration}

The high velocity of a low Earth-orbiting satellite such as Aura means that, while the scanning mirror physically rotates through the whole profile, a significant geographical distance will be traversed by the satellite. Figure 1 of Ern et al. (2011) illustrates this effect, as does our Fig. 4b.

We can make an estimate of the effect of this upon our measurements. Aura completes 14.6 orbits a day and HIRDLS takes around $15.5 \mathrm{~s}$ to perform a vertical scan. Accordingly, in the time taken to perform a complete vertical scan, the HIRDLS measurement track will have advanced by

$\Delta X=\left(\frac{2 \pi \times R \times 14.6}{24 \times 60 \times 60} \times 15.5\right) \mathrm{m}$,

where $R$ is the radius of a small circle around the Earth offset by $47^{\circ}$ from the great circle around the poles, $R \sim$ $6.4 \times 10^{6} \cos \left(47^{\circ}\right) \mathrm{m}=4.3 \times 10^{6} \mathrm{~m}$, giving a distance travelled during each scan of $\sim 72 \mathrm{~km}$.

A full vertical scan runs from the surface to a height of $\sim 121 \mathrm{~km}$, and hence the horizontal distance along-track between individual height levels (i.e. after the $1 \mathrm{~km}$ vertical interpolation) is approximately $0.6 \mathrm{~km}$. Accordingly, between the 15 and $80 \mathrm{~km}$ levels, the tangent point of a measurement will differ horizontally by $\sim 40 \mathrm{~km}$, producing a difference in $\Delta r_{i, i+1}$ of as much as $\Delta X=55 \%$ in some profile pairs at high altitudes relative to the geolocation height at $30 \mathrm{~km}$. This is a larger separation than the instrument weighting functions in the narrower direction, and is hence significant. We compensate for it in our analysis by scaling the profile separation distance $\Delta r_{i, i+1}$ appropriately for each height level before calculating $k_{h}$ and $M_{i, i+1}$, but this means that the along-track horizontal resolution limit varies with height due to the scan direction of the profiles. Figure $4 d$, discussed in greater detail below, illustrates this effect.

This scanning effect will in principle affect vertical wavelength measurements, since the vector of the instrument scan lies at $\sim\left(90^{\circ}-\arctan (1 / 0.6)\right)=31^{\circ}$ to the vertical. However, this is compensated for in the retrieval, which splines the measured radiances onto a regular vertical grid.

The high velocity of the satellite allows us to consider observed waves as having been measured effectively instantaneously (Ern et al., 2004).

\subsection{Direction of propagation}

Our measurements represent only the component of the signal lying along the satellite's travel vector. Due to the low probability of the horizontal wave vector lying along this direction, our measurements will tend to underestimate the true value of $k_{h}$, especially when there is a large angle between the true propagation direction and the measurement direc- tion. If waves tend to propagate zonally rather than meridionally, this will particularly affect measurements when the satellite is travelling in a mostly meridional direction, i.e. near the Equator, and have the smallest impact when the satellite is travelling more zonally near the turnaround latitudes $\left(\sim 62.5^{\circ} \mathrm{S}\right.$ and $\left.\sim 80^{\circ} \mathrm{N}\right)$. This effect is seen strongly in our results, and is discussed where appropriate.

\subsection{S-Transform limitations}

Our S-Transform analysis method inherently introduces further errors into the analysis. A range of sensitivity studies using perfect wave packets were carried out by Wright (2010), and can be summarised as follows. These limitations apply generally to S-Transform data.

1. Provided the signal is above the noise level of the data, the error on the measured temperature perturbation does not depend directly on the magnitude of the "true" temperature perturbation.

2. The error on the measured temperature perturbation is inversely proportional to the number of full wave cycles of the signal visible in the vertical direction: the greater the number of wave cycles, the more accurate the measurement. An insufficient number of wave cycles to fully resolve the signal will always reduce the measured temperature perturbation, and not increase it.

3. The error on the measured temperature perturbation depends upon the vertical wavelength of the signal; again, errors introduced in this way will only reduce the measured signal strength.

4. The error in the phase difference measurement, and hence $k_{h}$, due solely to limited vertical resolution, is less than $25 \%$ for wavelengths between once and twice the vertical resolution limit and less than $10 \%$ above this.

5. The error in the vertical wavenumber measurement is typically less than $\sim 10 \%$, provided at least one full cycle of the signal is observed; if less than one full cycle is observed, the measured vertical wavenumber will be smaller than the true value, by up to $\sim 20 \%$ for waves where only half a cycle is observed, and increasing rapidly below this level. This will especially affect long wavelengths at the top and bottom of the analysis, which will be edge-truncated and hence shifted downwards in apparent wavenumber.

To summarise, in addition to any uncertainties due to the actual measurements, we expect our analysis to systematically underestimate temperature perturbations, potentially by a very large proportion, in all conditions, and to generally underestimate vertical wavenumber in any conditions where we do not detect one or more full cycles of the same wave. 


\subsection{Aliasing}

An important limitation is the ambiguity of phase cycle in our estimates of $\Delta \phi$; that is to say, we cannot know purely from our measurements whether the measured phase difference of a $\zeta$ between two adjacent profiles represents $\Delta \phi_{\text {true }}$, $\Delta \phi_{\text {true }}+2 \pi, \Delta \phi_{\text {true }}+4 \pi$, etc. This is referred to as aliasing (Preusse et al., 2002; Ern et al., 2004), and will cause us to underestimate $k_{h}$ for large-wavenumber features in our data, perhaps very significantly. The effect of this on our results will be to redistribute these aliased waves across the measured wavenumber range. Wright and Gille (2013) suggest that a large proportion of the additional smaller-scale waves detected by our method may be aliased in this way.

If we assume (Ern et al., 2004) that such aliased waves have a random measured phase difference $\Delta \phi_{i, i+1}$, then this will distribute them evenly across the measured $k_{h}$ space (Eq. 1). In principle, a correction factor may be applied to account for this aliasing (e.g. Ern et al., 2004); however, such corrections make inherent assumptions about the spectral shape of the original wave distribution, and accordingly we do not use them here.

\subsection{Momentum flux calculation}

The derivation of Eq. (2) assumes that the waves under consideration can be described by the midfrequency approximation. This has been shown by Ern et al. (2004) to account for around a $10 \%$ difference between real and calculated values of $M_{i, i+1}$ for the CRISTA instrument.

\section{Global-mean wavenumber distributions}

\subsection{Vertical wavenumber}

Figure 1 shows the global distribution of the number of observed waves, as a function of the base-10 logarithm of vertical wavenumber $k_{z}$. Corresponding vertical wavelengths are provided on the top axis of the figure as a guide.

We first consider Fig. 1a. This shows the distribution at six height levels. At all heights, we see a broadly similar distribution, with larger numbers of observed waves at smaller wavenumbers, and a steady drop with increasing wavenumber. In particular, the number of observed waves drops by a factor of $\sim 6$ between $k_{z}=10^{-4.2}$ and $10^{-3.5} \mathrm{cpm}$.

Heights above $55 \mathrm{~km}$ (orange and brown lines) are truncated at a vertical wavenumber of $10^{-3.6} \mathrm{cpm}(4 \mathrm{~km}$ vertical wavelength) due to the reduced vertical resolution at these altitudes; the remaining lines continue to $10^{-3.3} \mathrm{cpm}(2 \mathrm{~km}$ vertical wavelength). This truncation is introduced because, although vertical features smaller than this are "detected" by the analysis, they are clearly spurious due to the nature of the retrieved product, the resolution of which drops by a factor of $\sim 2$ in this region.
Fewer small-wavenumber waves are observed at the 20,60 and $70 \mathrm{~km}$ altitude levels; this is due to the proximity of the vertical ends of the data set at 80 and $15 \mathrm{~km}$, which significantly reduces the possibility of properly observing a long vertical wave here. We also observe four subpeaks, centred on wavenumbers $10^{-3.34}, 10^{-3.47}, 10^{-3.64}$, and $10^{-3.95} \mathrm{cpm}$ (the last only weakly visible, very broad, and shifting with height value given is for $\sim 50 \mathrm{~km}$ altitude).

\section{High vertical wavenumber subpeaks}

The subpeaks observed in Fig. 1a are markedly different from the surrounding distribution, and consequently do not appear to be geophysical. Equivalently analysed SABER data (Fig. 1b) do not show any such subpeaks, with the exception of a subpeak at $\sim 10^{-3.64} \mathrm{cpm}$, which is very close to the resolution limit and thus may be due to aliasing of shorter waves into the observational filter of the instrument. The highestwavenumber subpeak in the HIRDLS data is proportionately larger than the others, and may be partially due to this effect. Additionally, analyses using high-resolution HadGEM analyses (not shown) sampled as HIRDLS data and analysed in the same way also show a distribution of the same form but without these subpeaks. Consequently, it is likely that the observed subpeaks are primarily non-geophysical. Figure 2 investigates this further.

Figure $2 \mathrm{a}$ shows, for a range of vertical wavelengths, the projection of a wave observed in the atmosphere at a range of heights onto the HIRDLS primary mirror elevation scan angle, computed as

$R_{\mathrm{E}}+H_{\mathrm{t}}=R_{\mathrm{S}} \cos \left(\epsilon_{\mathrm{LOS}}\right)=R_{\mathrm{S}} \cos \left(2 \epsilon_{\mathrm{M}}\right)$,

where $R_{\mathrm{E}}$ is the radius of the Earth, $H_{\mathrm{t}}$ the height of the instrument scan tangent point above the surface, $R_{\mathrm{S}}$ is the orbital height of the satellite relative to the centre of the Earth, $\epsilon_{\mathrm{LOS}}$ is the instrument scan angle, and $\epsilon_{\mathrm{M}}$ is the mirror angle, which must be multiplied by 2 to include the reflection from the mirror when calculating $\epsilon_{\mathrm{LOS}}$. In particular, this figure shows that features of wavenumber $k_{z}=10^{-3.95} \mathrm{cpm}$, i.e. $\lambda_{z} \sim 9 \mathrm{~km}$, will correspond to an elevation scan angle range of $\sim 0.09^{\circ}$, and that our other peaks at $k_{z}=10^{-3.64} \mathrm{cpm}$, $k_{z}=10^{-3.47} \mathrm{cpm}$ and $k_{z}=10^{-3.34} \mathrm{cpm}$ correspond to integer ratios of this wavelength $\left(\lambda_{z} \sim 4.5,3\right.$ and $2.25 \mathrm{~km}$ respectively).

Figure $2 \mathrm{~b}$ meanwhile shows the time variation of the peak centred at $k_{z}>10^{-3.64} \mathrm{cpm}$, normalised to the value of the distribution at $k_{z}>10^{-3.53} \mathrm{cpm}-$ at the latter point, the distribution appears close to a linear fit from the higher wavenumbers, and is thus assumed to be broadly representative of the "background" to the anomalous peaks. The black horizontal line shows the expected value of this normalised distribution at $k_{z}>10^{-3.64} \mathrm{cpm}$ if the data were interpolated linearly across the range with the feature removed. Three different height levels are shown, chosen from the middle of the data coverage range to avoid any possible edge truncation ef- 

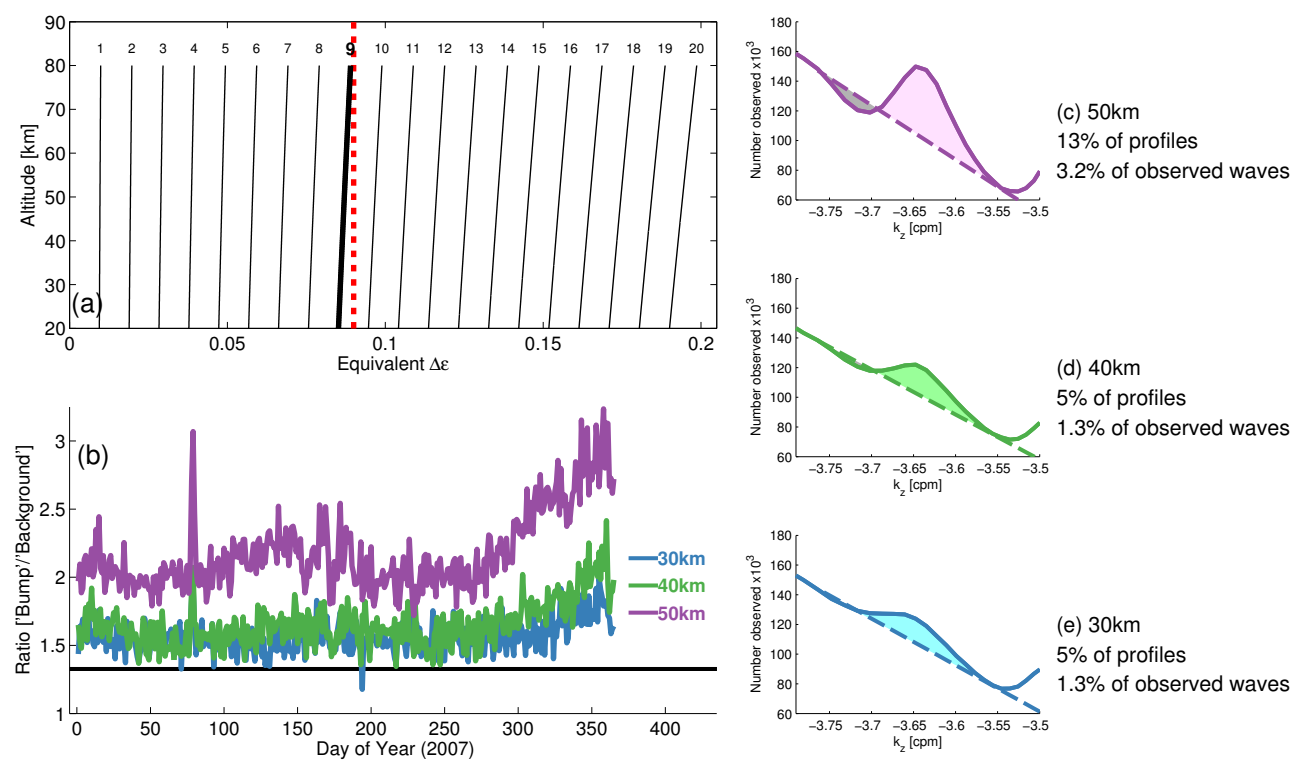

Figure 2. (a) Equivalent projection on the instrument primary mirror elevation angle of a feature of a wavelength indicated by numerical values at the top of each line, as a function of altitude. Grey dashed line indicates approximate wavelength (in elevation angle space) of blockage-induced oscillations illustrated in Fig. 5 of Gille et al. (2008); bold line indicates nearest calculated wavelength to this angle. (b) Time series of the ratio between the observed number of waves at $\log _{10}\left(k_{z}\right)=-3.653$ (peak of anomalous bump) and $\log _{10}\left(k_{z}\right)=-3.530$ (trough between two peaks, where data approximate a linear fit to the distribution) for daily global-mean data at three height levels. Horizontal solid line indicates the ratio that would be observed if data were extrapolated linearly over the anomalous region. (c-e) Close-up illustration of the peak at $\log \left(k_{z}\right) \sim-3.65$ for three height levels, illustrating the difference between the feature at each height (solid line) and a linear fit across the region (dashed lines). Coloured shaded region indicates positive anomaly; grey shaded region indicates region of possible wave undersampling, discussed in the text.

fects. We see that, in general, the size of the feature co-varies at all three levels, with the amplitude of the feature increasing with height. Separate analyses (omitted for brevity) further show that the feature does not vary systematically as a function of latitude, height range of data supplied to the STransform analysis, or wavelet size used; thus, it is unlikely that the feature arises from the analysis methodology. Taken together, these two figures suggest a possible explanation in terms of the instrument blockage.

As shown in Fig. 5 of Gille et al. (2008), the uncorrected HIRDLS data from the instrument exhibit strong horizontal features in the measured radiance at a characteristic "wavelength" on the instrument focal plane corresponding to an elevation scan angle $\sim 0.09^{\circ}$, assumed to be due to resonances in the Kapton blockage set up by contact with the mirror. As seen in Fig. 2a, this corresponds to an observed wavelength $\sim 9 \mathrm{~km}$. The other observed peaks would then correspond to aliased near-multiples of this.

Validation exercises have previously suggested that this feature was successfully suppressed to below the level of the instrument noise by the correction and retrieval processing chain, but it is possible that some of this signal remains in the data. Since our gravity wave detection methodology (Sect. 3) examines the data for co-varying features in profiles, this will tend to select strongly for any such variation remaining after processing, as the (uncorrected) blockage-induced signal will co-vary between profiles much more strongly than any true geophysical signal.

Figure $2 \mathrm{c}-\mathrm{e}$ attempt to estimate the contribution to the observed spectrum arising due to this issue. Each panel shows, for the same three altitude levels as Fig. 2b, a zoomed-in region of Fig. 1a, focusing on the anomalous peak centred at $k_{z}>10^{-3.64} \mathrm{cpm}$. In each case, we linearly interpolate across the anomalous region, and use this fit to estimate the number of additional signals contributed by the peak, indicated by the coloured shaded region. These estimates, computed by estimating their integrated area to the total area below the respective curves, suggest that between 5 and $13 \%$ of profiles (depending on height) are affected by this peak, which due to the detection of overlapping presumably geophysical signals corresponds to $1.5-3 \%$ of observed wavelike signals. Since there are four such peaks, this gives an approximate upper bound of $20-50 \%$ of affected profiles and $6-12 \%$ of observed waves; this is likely to be a significant overestimate, since it assumes that each of these peaks is caused by entirely independent signals, whereas in practice the feature is likely to appear at multiple wavelengths in the same profile. Although this number is large by number of observations (Fig. 1), the spurious features are typically small in temperature amplitude and in terms of apparent momentum flux 
transported by the "waves" they represent (see e.g. Figs. 5 and 8 , discussed below).

Our results further suggest (Fig. 2c) that some real waves at close wavenumbers may be masked by these features. The purple shaded region shows our estimate of the positive anomaly (i.e. spurious additional waves) at this wavelength at $50 \mathrm{~km}$ altitude; the grey shaded region, meanwhile, shows an apparent deficit of observed waves at a slightly smaller wavenumber when compared to a linear fit. Since the detection method is based on the analysis of peaks in a spectrum, it is thus likely that the spurious peaks in many profiles are "drowning out" the true spectral peaks at slightly smaller wavenumbers in profiles where such waves exist. This may particularly be the case at wavenumbers $\sim 10^{-3.85} \mathrm{cpm}$. Here, we have comparatively few spectral points, and observe a distinct "wiggling" of the observed spectrum: this is consistent with a spurious peak at $9 \mathrm{~km}$ wavelength affecting the true distribution around it to some degree.

Other methods of detecting gravity waves in HIRDLS data (e.g. Alexander et al., 2008; Ern and Preusse, 2012) have selected only for the one or at most two largest-amplitude signals in each profile, which may explain why this effect has not been noted previously. To test this, Fig. 1c illustrates the results that would be obtained using the singlepeak (HIRDLS-pk) method. We see no such anomalous signal; this is partially due to the complete lack of signals at high $k_{z}$, but the observed distribution includes the peak at $10^{-3.95} \mathrm{cpm}$ at which one of the peaks would be expected to occur, suggesting that in the majority of observed cases another, presumably geophysical, signal dominates over this effect. The features will also have been hidden in the previous two studies using the current method, in Wright and Gille (2013) by the large bin size in $k_{z}$ at high wavenumbers and in Wright et al. (2013) by the small momentum flux impact of this effect.

Since the majority of our following analysis focuses upon the observed wave spectrum decomposed as a function of both $k_{h}$ and $k_{z}$, it is difficult to remove these features. For example, a simple downscaling of the number of observed waves in the regions centred on these peaks would be inaccurate, and hard to implement: in Fig. 5 (discussed below), we see that these peaks appear to spread across all horizontal wavenumbers rather than to be focused at a particular range, and thus any scaling-down of the number of observations at these vertical wavenumbers would be faced with the additional task of identifying them in this second dimension. The time and height variation of the features (Fig. 2b) provides a further stumbling block to their removal. Finally, the temperature perturbation amplitudes of the signal are not easily distinguished from the overall spectrum. Accordingly, we do not remove these data from our analyses, but instead include them and address them directly where necessary. We do, however, attempt to mitigate the effect by only analysing data at vertical wavelengths shorter than $16 \mathrm{~km}$; due to the spacing of output bins from the ST analysis, all bins at longer vertical wavelengths will include at least one peak due to this effect, with no inter-peak gaps in the distribution allowing us to assess the relative contribution of the contaminating peaks.

\subsection{Horizontal wavenumber}

Figure 3 shows equivalent results for horizontal wavenumber $k_{h}$. We observe a distribution which rises as a function of horizontal wavenumber. A flattening of the distribution is observed at intermediate wavenumbers, $k_{h} \sim 10^{-6.1}-k_{h} \sim$ $10^{-5.6} \mathrm{cpm}$; this appears to be due to the bias in observed horizontal wavenumber at equatorial latitudes due to the meridional path of the satellite (Sect. 4.4), and is discussed further in Sect. 6. Aside from this flattening, the data otherwise rise consistently until a peak is reached at $k_{h}=10^{-5.35} \mathrm{cpm}$.

Above $k_{h}=10^{-5.35} \mathrm{cpm}$, a discontinuity is observed, with the absolute peak followed by a sudden drop and then by a secondary peak at $k_{h}=10^{-5.25} \mathrm{cpm}$. This arises due to the instrument scanning pattern (Sect. 4.3), and is explained by Fig. 4, discussed below.

A general trend is seen of the distribution shifting towards higher $k_{h}$ with height; this will be discussed below.

Figure $3 b$ and $c$ show equivalent results for SABER and the HIRDLS-pk method. The HIRDLS-pk results show a distribution falling off at horizontal wavenumbers greater than $\sim 10^{-6.7} \mathrm{cpm}$ at $20 \mathrm{~km}$ altitude, with the turning point in $k_{h}$ increasing with altitude; this suggests that the additional waves contributed by the method of Wright and Gille (2013) may include significantly more short horizontal waves. Note, however, that it is difficult to ascertain the full effects of noise on our measurements. While the method is designed to mitigate against the inclusion of instrumental noise via the co-varying amplitude methodology and the noise-floor comparison, Ern et al. (2004) suggest that random fluctuations would peak at around $4 \times$ the horizontal sampling distance, and increase with altitudes. Since we see these effects in all three data sets (HIRDLS, HIRDLS-pk and SABER), they may contribute to our distributions.

The SABER results, meanwhile, show a distribution with a form very similar to that of the primary HIRDLS results. This suggests that the anomalous blockage-induced peaks do not have a preferential apparent horizontal wavenumber, but are instead distributed across the whole observed wavenumber range.

\section{High horizontal wavenumber discontinuity}

Figure 4a shows the full observed $k_{h}$ distribution, reproducing Fig. 3. Figure 4b, meanwhile, illustrates the instrument scanning pattern for all observations used in this study. The instrument scans up and down repeatedly (blue lines) as it travels along the observational track (horizontal axis); since the top of the scan is a large vertical distance from our observation levels, whereas the bottom of the scan is comparatively close, this results in a characteristic alternating pattern 

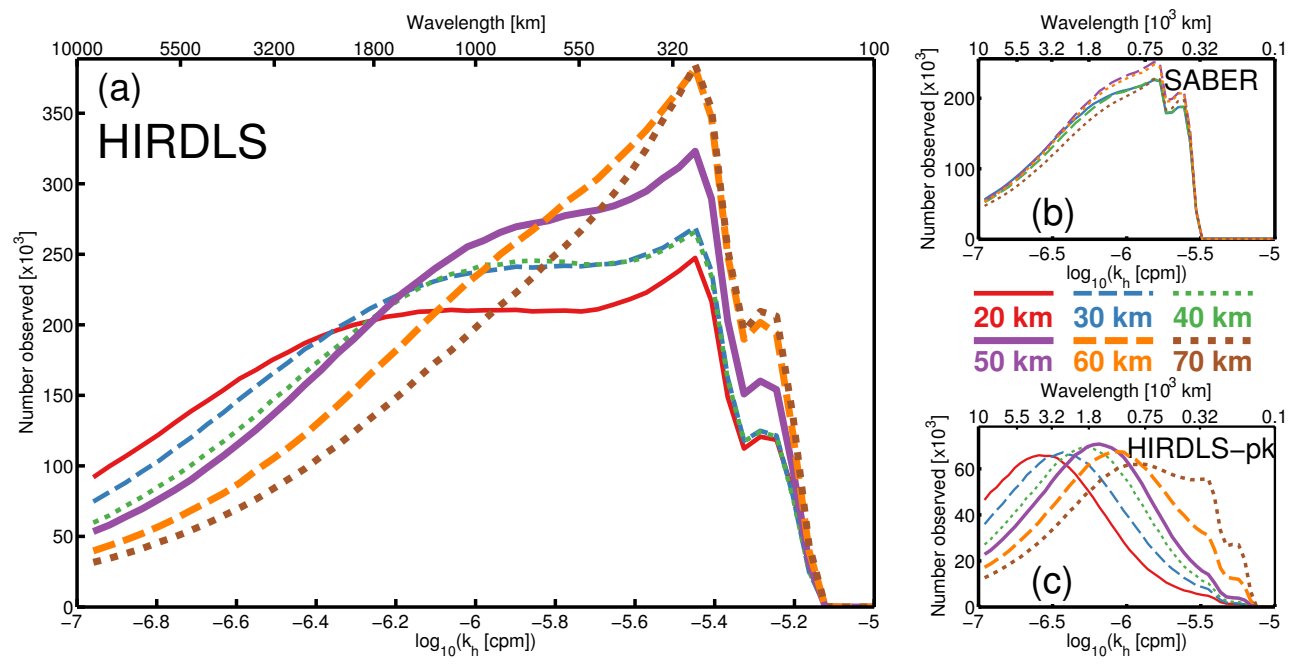

Figure 3. As Fig. 1; horizontal wavenumbers.
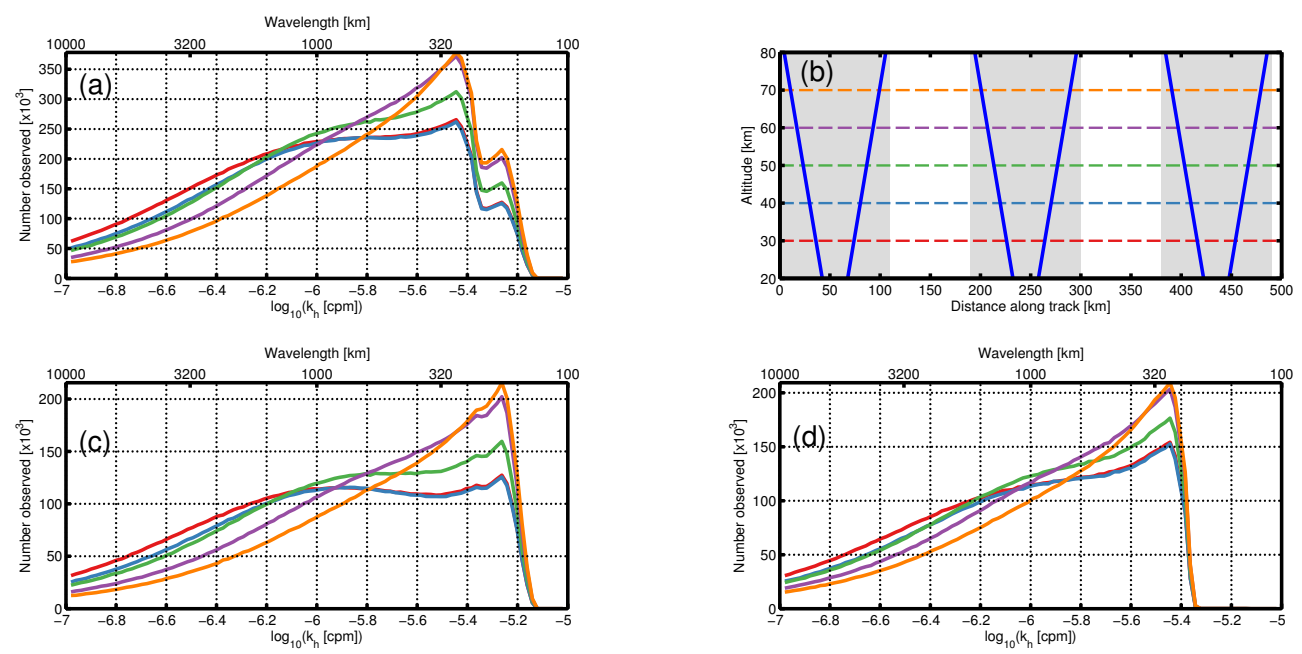

Figure 4. (a) Observed $k_{h}$ distribution for all observations, reproducing Fig. 3. (b) Illustration of instrument scanning pattern; blue lines indicate sequential instrument scans, horizontal dashed lines indicate height levels shown in panels (a, c, d), and shaded (unshaded) regions indicated closely spaced (widely spaced) pairs. $(\mathbf{c}, \mathbf{d})$ Distributions for (c) closely spaced and (d) widely spaced profile pairs only.

of closely spaced (highlighted in grey) and widely spaced profile pairs. Since the measurable $k_{h}$ depends strongly upon the distance between profile pairs (Sect. 3), this imposes a different minimum observable wavelength for the closely spaced and widely spaced pairs.

To confirm this, Fig. 4c and d show, respectively, separate distributions for closely spaced profile pairs only and widely spaced profile pairs only. In both cases, we see a hard cutoff at the horizontal resolution limit, corresponding to the peak of each distribution. Since above $k_{h} \sim 10^{-5.35}$ only closely spaced profile pairs can contribute to our distribution, we see a sharp dropoff and secondary peak in the combined result.

To avoid this issue affecting our results, we omit widely spaced profile pairs from our analysis. This halves the number of useful observations, but provides greater consistency and a finer resolution limit without the need to correct for this effect.

\section{Joint wavenumber analyses}

\subsection{Global mean}

Figure 5a shows the distribution of observed waves as a function of both $k_{h}$ (horizontal axis) and $k_{z}$ (vertical axis), at the $32 \mathrm{~km}$ altitude level. This level is chosen as it is approximately the lowest height level at which no detected wave signals could be edge-truncated (tropopause plus $16 \mathrm{~km}$ ). It should be noted that this global-mean distribution is averaged 

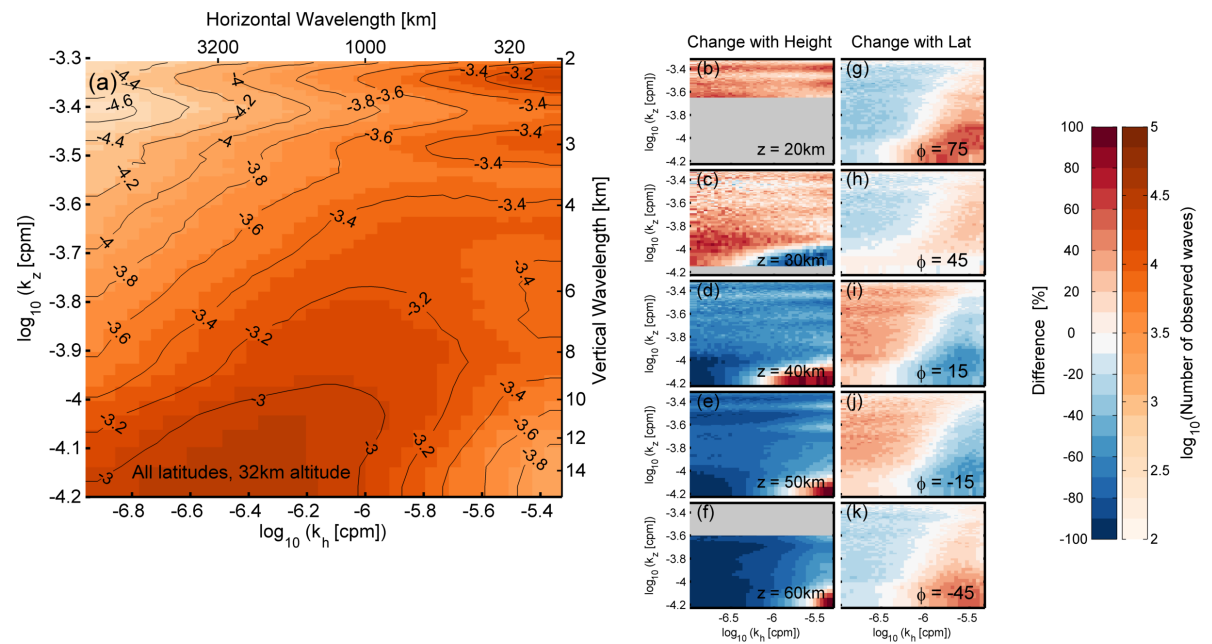

Figure 5. (a) Global mean annual-mean distribution of the fraction of observed waves as a function of horizontal and vertical wavenumber at $32 \mathrm{~km}$ altitude. (b-f) differences from this distribution at five height levels, specified in the panel. (g-k) differences from this distribution for five latitude bands, centred on the latitude specified.

over a vast range of geophysical regimes, and accordingly is meaningful as a reference only.

We see that the observed numerical distribution is dominated by waves with small horizontal and vertical wavenumbers, i.e. with long horizontal and vertical wavelengths, with the number of observed waves in bins in this region (bottom left to bottom centre) typically 2 or more orders of magnitude above the numbers in the least-observed region at top left. This will at least partially relate to observational effects, rather than to the geophysical distribution of such waves; a wave with a longer wavelength in either direction is likely to have a larger physical extent, and is thus more likely to be observed in a measurement profile randomly located in the vicinity of the wave. Additionally, such waves are likely to induce larger temperature perturbations (Fig. 8b) and thus are more likely to be above instrument noise levels. Furthermore, as shown by Fig. 4 of Preusse et al. (2002), the temperature perturbations of such waves will tend to be more easily detectable due to high instrument sensitivity. Duplication effects are compounded in the horizontal direction: as discussed above, it is highly non-trivial to distinguish between the same horizontal feature in adjacent profiles, and consequently long horizontal waves may well appear in several profiles, particularly when the satellite is travelling in a similar direction to the wave vector. Future refinements of the analysis method will investigate this further, using methods based upon the Fourier uncertainty principle.

The smallest number of observed waves lies in the top left of the plot, i.e. large vertical wavenumber and small horizontal wavenumber. As discussed above, the paucity of observations here in the vertical domain may well be due to random-sampling considerations; however, bias in observed horizontal waves would tend to increase rather than decrease the number of observed waves here, and detectability of their temperature perturbations should be reasonable (although the amplitude of such waves may be low). As a result, the small number of waves observed in this region is likely to be a real effect, suggesting that the atmosphere may support comparatively few short-vertical-long-horizontal-wavelength "pancake" waves.

We see the anomalous, presumably blockage-induced, spikes observed in Fig. 1 most strongly at top right; these do, however, extend across most of the horizontal range to at least some degree. Beneath these peaks, we continue to see a much higher number of waves than in the top left or bottom right, suggesting that even without the peaks this is a significant contributing region to the overall distribution. Finally, at bottom right, we see a region of few waves; this is consistent with the strongly reduced detectability at this combination of wavelengths. Detectability here should be by far the worst of any part of the 2-D spectrum; thus, the larger number of signals observed here than at, for example, the top left, may suggest that the atmosphere can support many short-horizontal-long-vertical-wavelength waves.

An apparent discrepancy is seen between our results and the HIRDLS-derived momentum flux wavenumber distributions of Ern and Preusse (2012). There, a clear peak was seen in tropical waves at $k_{h}=10^{-5.75} \mathrm{cpm}$ and $k_{z}=10^{-3.90} \mathrm{cpm}$. However, their method selected only the two largest signals in each rolling $10 \mathrm{~km}$ window, and accordingly will not have included the shorter waves that make up a large part of our distributions, hence producing results with a very different final form, more similar to our HIRDLS-pk analyses. As shown in Figs. 1c and 3c, this data set peaks at much longer vertical and horizontal wavelengths than HIRDLS-all, explaining the majority of this discrepancy. Additional differences remain in the precise location of the peak, which lies (at the $30 \mathrm{~km}$ level, i.e. the closest analysed height level 
to their analysis) at $k_{h} \sim 10^{-6.4} \mathrm{cpm}$ and $k_{z} \leq 10^{-4.2} \mathrm{cpm}$ in our HIRDLS-pk distribution. The difference in $k_{h}$ peak location arises due to the strong dependence of observed MF on wavenumber (Eq. 2). Part of the difference in $k_{z}$ peak location is explained by the slightly larger- $k_{z}$ waves observed near the Equator (e.g. Fig. 5i and j) relative to other latitudes, but this cannot account for all of the differences, which may instead arise due to the very different analysis methods used.

Subsequent panels of this figure (Fig. 5b-k) are shown as percentage differences from this $32 \mathrm{~km}$ global distribution, with colours representing the percentage difference in the proportion of total observed waves at a given $\left(k_{h}, k_{z}\right)$. This is a slightly complicated normalisation, but is chosen due to the small differences in visual appearance between undifferenced distributions; it should be noted that the distribution appears broadly identical at all heights when shown un-differenced ${ }^{2}$.

\subsection{Height variations}

Figures 5b-f show the difference between the observed distribution at $32 \mathrm{~km}$ and that at five other height levels. Greyshaded regions in individual panels indicate areas of the spectra which may experience edge truncation at that level.

We see two key trends, with increasing height leading to (i) larger horizontal and (ii) smaller vertical wavenumbers. The first such difference is clearly visible in Fig. 3 for all three data sets. The latter is harder to see in the absolute observed values seen in Fig. 1, and only becomes apparent when the data are normalised at each level individually; as discussed above, at least part of this change with height may be associated with noise effects. The change in vertical wavelength is consistent with previous observations dating back decades (e.g. Fritts, 1984, and references therein).

\subsection{Latitudinal variations}

Figures $5 \mathrm{~g}-\mathrm{k}$ show the differences from the global mean in Fig. 5a for the same analysis performed on specific $30^{\circ}$ latitude bands centred on the latitude indicated in the panel, all at the same $32 \mathrm{~km}$ altitude level. Note that the northerly limit to observations is at $80^{\circ} \mathrm{N}$, and thus that panel $\mathrm{g}$ only represents the range $60-80^{\circ} \mathrm{N}$.

We see very clear differences, with a bias towards smaller horizontal wavenumbers near the Equator (between Fig. 5i and $\mathrm{j}$ ) and towards larger horizontal wavenumbers at higher latitudes. As discussed in Sect. 4.4, this is at least partially due to the polar orbit of the instrument, which leads to the satellite travelling near-meridionally near the Equator, combined with Coriolis parameter effects which allow a broader range of wave-intrinsic frequencies near the Equator (Preusse et al., 2006). If we assume a zonal bias to the

\footnotetext{
${ }^{2}$ Compare e.g. Fig. 5g-k with Fig. $6 \alpha-\epsilon$, which show the same data, with this normalisation in the former case and that used in Fig. 5a in the latter.
}

true wave field, observations here will tend to significantly over-measure the distance between phase fronts due to the geometry of the scan, and consequently significantly underestimate $k_{h}$. There is some difference in the $k_{z}$ direction, with smaller $k_{z}$ at higher latitudes and larger $k_{z}$ near the Equator, but this effect is smaller than the $k_{h}$ effect; this is consistent with e.g. Alexander et al. (2008), Yan et al. (2010) and Ern et al. (2011).

Aside from this, minimal differences are observed between these distributions. This is largely due to the $k_{h}$ effect drowning out such variation visually. To compensate for this, all following analyses will be normalised for latitude by comparing only within a given latitude band or a given region.

\section{Regional variations}

A large part of the remainder of this study will focus on regional variations in these above distributions. Figures 6-7 and 9-10 accordingly use a fixed set of regions, identical in each case. In each of these figures, each region (panels a-ad) is a $30^{\circ}$ latitude by $60^{\circ}$ longitude box, with boxes spanning from $60^{\circ} \mathrm{S}$ to $90^{\circ} \mathrm{N}$ and $180^{\circ} \mathrm{W}$ to $180^{\circ} \mathrm{E}$. Note that data are not available poleward of $80^{\circ} \mathrm{N}$. Maps are plotted over these regions to aid interpretation; however, variations within each panel do not correspond to this subregional geography, but only to the distribution in the panel as a whole. Panels $\alpha-\epsilon$, the rightmost column, will show zonal means for the corresponding latitude band.

\section{Seasonal joint-wavenumber analyses}

Figures 6 and 7 show the observed $k_{h}-k_{z}$ distributions for each of our geographic regions for global summer (JJA NH (Northern Hemisphere), DJF SH (Southern Hemisphere)) and winter (DJF NH, JJA SH) respectively. The panels are normalised in a similar way to Fig. 5b-k above, but with the differences being not from the annual mean global mean, but from the annual mean zonal mean at that latitude (shown identically in Figs. $6 \alpha-\epsilon$ and $7 \alpha-\epsilon$ ). This allows us to focus on variations other than the instrument- and Coriolisparameter-induced variation in horizontal wavelength with latitude seen in Fig. $5 \mathrm{~g}-\mathrm{k}$, which would otherwise dominate all panels. Analyses were also carried out for spring and autumn, but have been omitted for brevity as the variations they showed were much smaller than for summer and winter.

We see the largest relative variations at low latitudes and in the bottom right of each panel, i.e. low $k_{z}$ and high $k_{h}$. Since wind-based spectral filtering in this region is primarily driven by the QBO, with a scale much longer than the year examined here, this may represent variation in the source mechanisms in this region, which is highly convectively active. Alternatively, it may indeed partially be due to QBO-related filtering, specifically the Doppler shifting of waves in and out of the observational filter of HIRDLS by the partial phase 


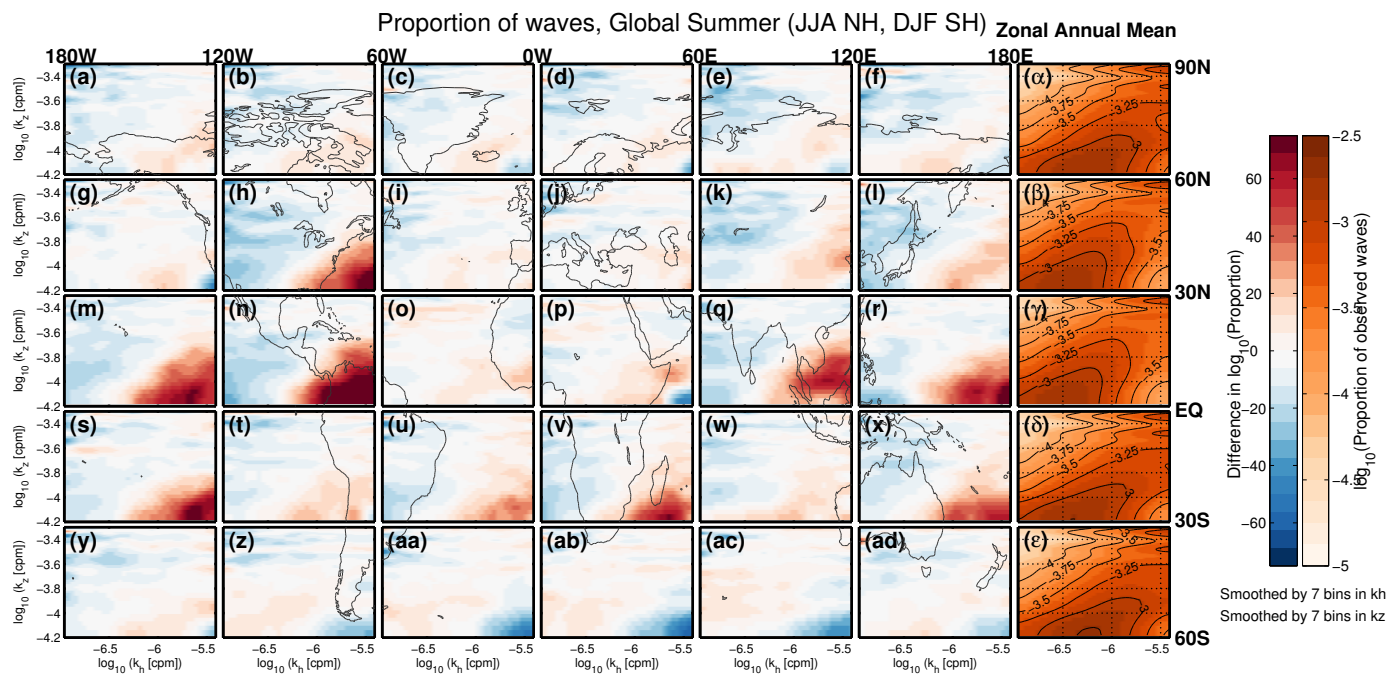

Figure 6. $(\boldsymbol{\alpha}-\boldsymbol{\epsilon})$ Zonal mean annual-mean distributions of the fraction of total observed waves for each latitude, as a function of $k_{h}$ and $k_{z}$. (aad) Equivalent distributions for each of our analysis regions in summer (JJA in the Northern Hemisphere, DJF in the Southern Hemisphere). Data are shown as differences from the corresponding zonal mean annual mean. A global map is overplotted for easy identification of geographic regions; note that the data shown in each panel are as a function of $k_{z}$ and $k_{h}$ only, and are not related in any way to the geography at scales below that of the region box. All values at $32 \mathrm{~km}$.

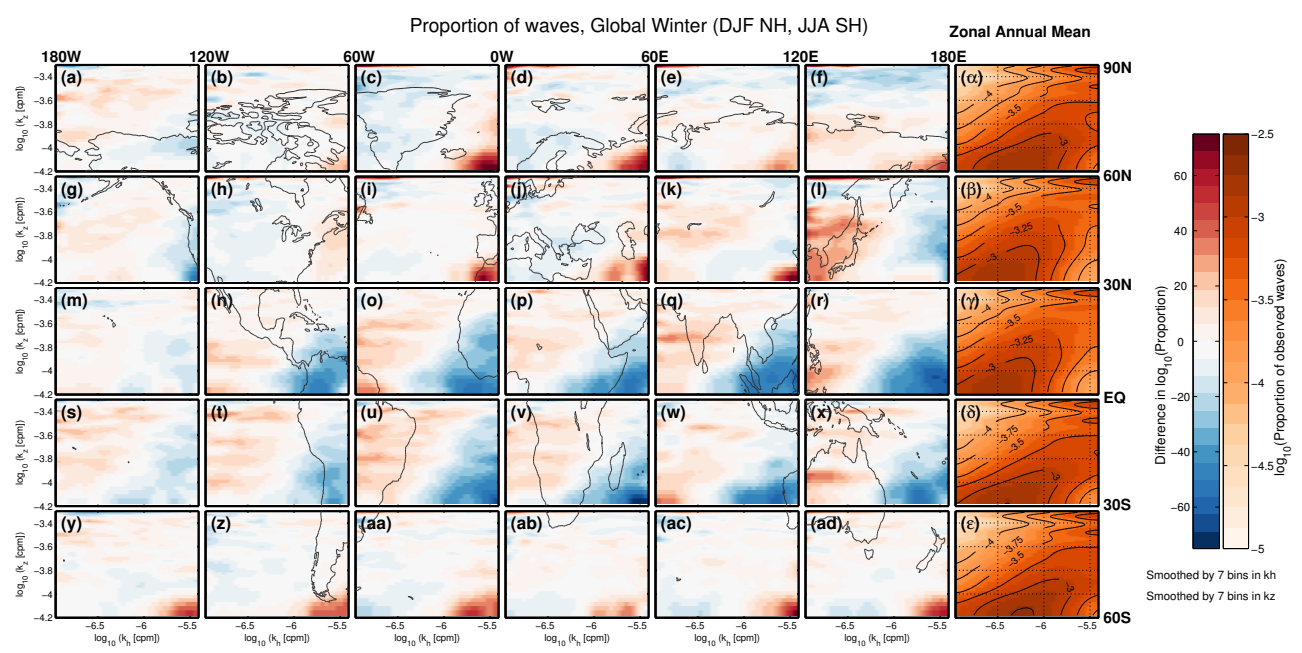

Figure 7. As Fig. 6 for global winter (DJF in the Northern Hemisphere, JJA in the Southern Hemisphere).

change of the QBO winds over the 6 months between Figs. 6 and 7. Examination of a longer period of time would help to elucidate this, but even the full 3 years of HIRDLS data are unlikely to provide a sufficiently long record to decouple this effect completely. Source changes are likely to be the dominant of the two factors due to the strong seasonality of convective activity in this region (e.g. Wright and Gille, 2011).

Smaller differences are seen at higher latitudes, and do not display such strong seasonal variation. In particular, at the highest latitudes, we often see an enhancement relative to the annual mean at many wavelengths in both summer and winter, i.e. large numbers in these seasons and low numbers in autumn and spring (not shown). Due to this behaviour, these regions are discussed below, where whole-year time series are shown.

\section{Relative variations of wave sub-species}

\subsection{Definitions and relative importance}

As we saw above, major differences between regions of the wavenumber distribution tended to manifest as peaks in the corners of each panel. Hence, it may be useful to subdivide our analysis by wavelength and to study separately the time 
evolution of these individual components of the distribution. Figure $8 \mathrm{a}$ accordingly divides the overall observed wave distribution into four distinct subtypes, or species, defined by wavenumber: short-vertical long-horizontal ("Sl”, top left), short-vertical short-horizontal ("Ss", top right), long-vertical long-horizontal ("Ll", bottom left), and long-vertical shorthorizontal ("Ls", bottom right).

Figure $8 \mathrm{~b}$ shows the mean temperature anomaly associated with each $k_{h}-k_{z}$ combination, indicating that the largest temperature perturbations are associated with species Ll. From this, one might initially conclude that waves of species $\mathrm{Ll}$ were the most important, due to their large amplitude - in particular, this implies a large potential energy per wave, $(1 / 2)(g / N)^{2}(\hat{T} / \bar{T})^{2}$. However, a vitally important geophysical quantity is the MF transported by the waves - in particular, this is one of the key parameters used in weather and climate modelling. In the mid-frequency approximation, this can be characterised by Eq. (2) above. There are three key variable terms in this which we can derive directly from HIRDLS data: $k_{h}, k_{z}$ and $\hat{T} / \bar{T}$, where $k_{z}$ and $k_{h}$ combine in the ratio $k_{h} / k_{z}$. For the waves we can observe with HIRDLS, this ratio can vary over nearly 3 orders of magnitude, as shown in Fig. 8c. As a consequence of this, the observed momentum flux per wave, Fig. 8d, is almost entirely dominated by Ls waves, particularly those at the very largest $k_{h}$ and smallest $k_{z}$, which as shown by Figs. 6 and 7 represent the bulk of the variability in our observations once $k-h$ variations due to orbital geometry and/or the variation of the Coriolis parameter with latitude are removed. Our results suggest, therefore, that variations in the number of observed Ls waves appear critically important to the variability of the global MF distribution in a much more fundamental way than the other three species.

As shown by Fig. 5, the global numerical distribution of observed waves is dominated by waves of species $\mathrm{Ll}$ and Ss. This numerical dominance of species Ll may be due in part to their larger mean temperature perturbations (Fig. 8b) and consequent easier detection in temperature data; however, this clearly cannot be the whole reason, due to the relatively small mean temperature perturbations for species Ss.

\subsection{Absolute and relative variations of observed species}

Figure 9 examines the time variation over the year 2007 for each of our four wave species, as a time series of the total number of observed waves per profile. The data exhibit significant day-to-day variability, and have been smoothed by 2 weeks to aid interpretation.

In general, the most-observed species is type Ss, with around 1.0-1.3 waves per profile (wpp) in most regions and at most times, whilst the least-observed species is type Sl, with typically $\sim 0.4 \mathrm{wpp}$. The former type includes a significant contribution from the anomalous subpeaks, which may contribute to the large number of observed signals, while the latter is difficult to detect due to limb-sounding sensitivity
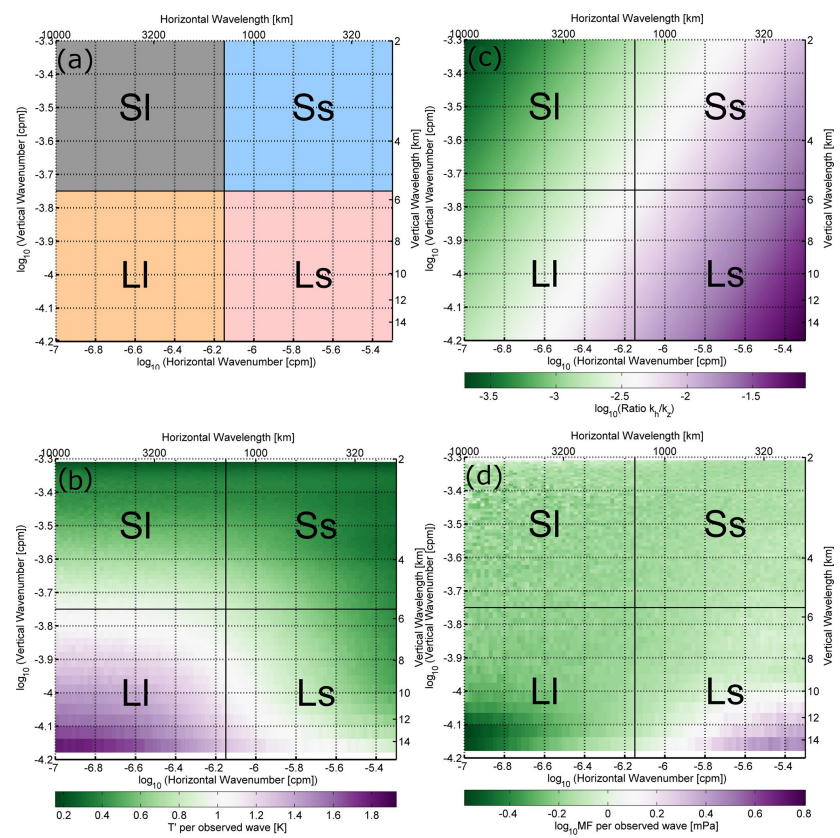

Figure 8. (a) Diagram indicating the four species of waves we define and examine. In terms of wavelength, these are short-vertical, short-horizontal (Ss, top right), short-vertical long-horizontal (Sl, top left), long-vertical short-horizontal (Ls, bottom right), and longvertical long-horizontal (Ll, bottom left). (b) Observed annualmean global-mean temperature perturbations per wave event, (c) ratio $k_{h} / k_{z}$, and (d) observed annual-mean global-mean momentum flux per wave event for analysed wavelength combinations. All values at $32 \mathrm{~km}$.

considerations (Preusse et al., 2002), which may explain the comparatively low number. However, this limitation applies more strongly to waves of type Ls, and thus cannot fully explain the difference. The number of observed waves of the two long-vertical species, Ls and Ll, in general lie between these values, with both $\mathrm{Ls}$ and $\mathrm{Ll}$ varying between around 0.4 and 1.4 wpp over the course of the year.

Figure 10, meanwhile, shows the same data, normalised such that the annual mean value for each species equals 100 . This emphasises the variability of each species with respect to time, and shows that, while in some regions and at some times all four species can vary together, at others different species can vary independently of each other, often with some apparent compensation between different species as one rises in observed frequency to take the place of another. The latter effect will be discussed further in Sect. 8.3.

Deviations from the mean are observed at Arctic latitudes in winter and spring, specifically between days 1 and 100 in Fig. 10c-f. The relatively low numbers during this time are consistent with filtering of waves by the polar vortex, and the variations coincide with vortex influences by large-scale planetary waves.

The next largest variations are those of type Ls, particularly at tropical and subtropical latitudes, i.e. Fig. $10 \mathrm{~m}-\mathrm{x}$, 


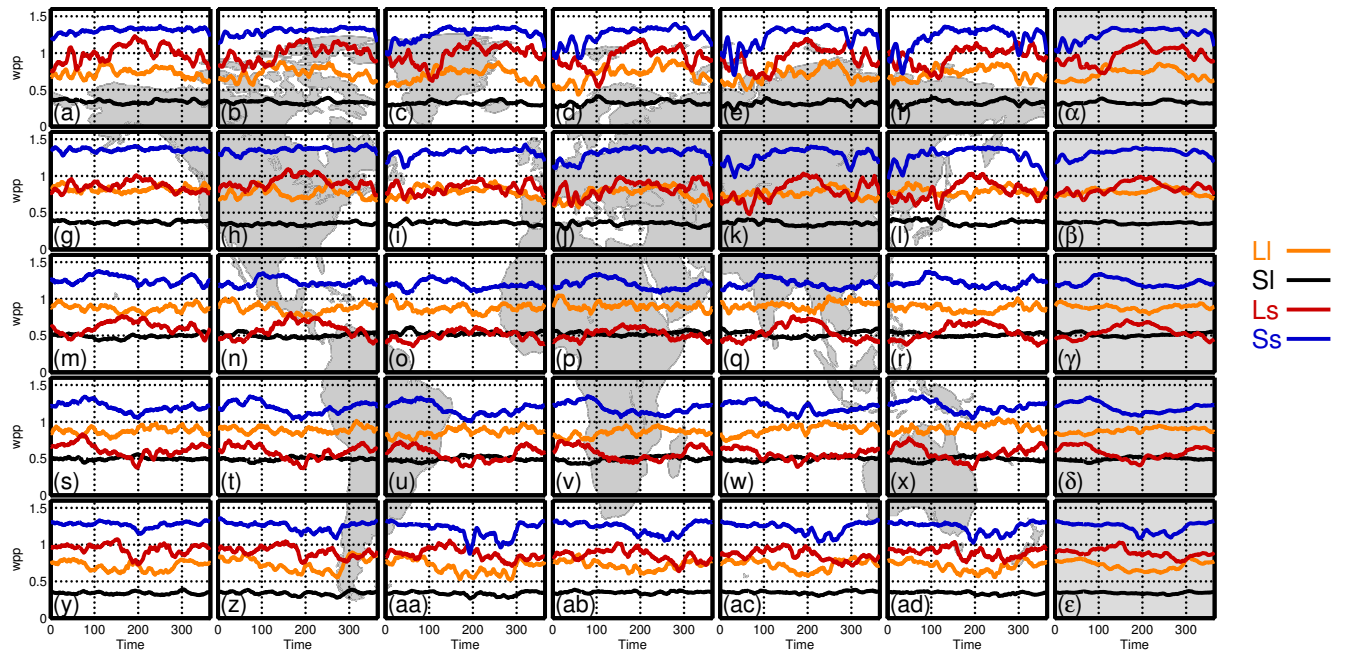

Figure 9. (a-ad) Regional time series showing the number of observed waves per profile (wpp) for each species. Note that wpp are defined as waves present at the analysis level, which does not necessarily correspond to the profile as a whole. Data have been smoothed by 2 weeks. $(\boldsymbol{\alpha}-\boldsymbol{\epsilon})$ Zonal mean time series. Units of time are calendar day from $1 \mathrm{January}$. All values at $32 \mathrm{~km}$.

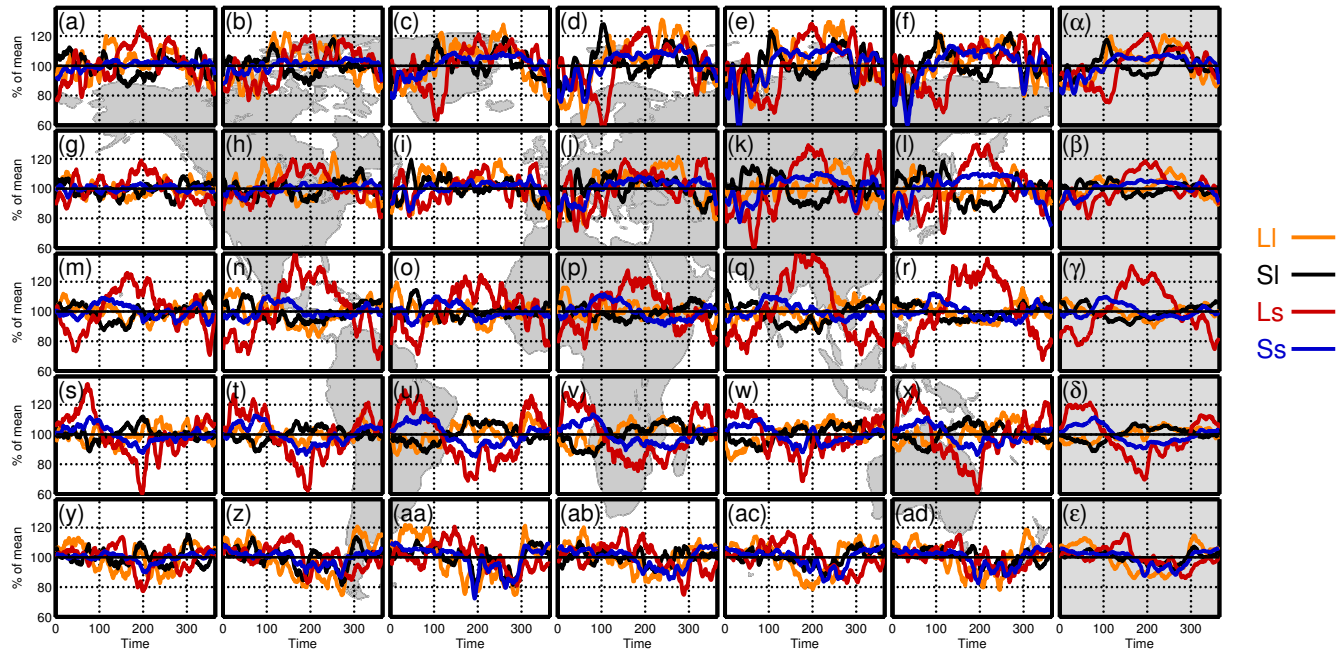

Figure 10. As Fig. 9 but normalised such that the annual mean for each species is equal to 100 .

$\gamma-\delta$. This has important consequences: as discussed above, this species is by far the dominant carrier of large momentum fluxes, and the large temporal variations of these species thus contribute significantly to the temporal variability of MF. In particular, we see large peaks in the observed number of Ls waves during monsoon periods in monsoon regions, i.e. Fig. 10n, p, q, r, $\gamma$ in NH summer and Fig. 10t, u, v, $\mathrm{x}, \delta$ in $\mathrm{SH}$ summer (Li and Zeng, 2002; Wright and Gille, 2011). This suggests that the large momentum fluxes previously observed to be associated with the monsoon (Wright and Gille, 2011) are dominantly carried by Ls waves; the correlation between our Ls wave time series and outgoing longwave radiation (OLR) over the particularly intense monsoon regions (panels $\mathrm{q}$ and $\mathrm{r}$ ), for example, is $\sim-0.7$. This is consistent with previous work, e.g. Jiang et al. (2004) and
Ern and Preusse (2012). The absolute number of observed Ls waves is not especially low compared to other regions at the same latitude during the other parts of the year (e.g. Fig. $9 \mathrm{~m}$, $\mathrm{o}$ in $\mathrm{NH}$ summer), suggesting that the relatively low value during the other parts of the year are a baseline rather than a reduction in Ls waves due to other processes.

Interestingly, comparatively little variation is observed in the relative distribution of all four species around the wellknown Andes/Antarctic Peninsula hotspot, in Fig. 10z-aa. This region has previously been observed to dominate the global MF distribution, with values in JJA typically an order of magnitude or more larger than the next largest peak at any other location or time. We see a reduction here in the number of observed waves in all four species during the period of enhanced MF, especially in Fig. 10aa; this reduction con- 
tinues downstream of the hotspot through Fig. 10ab-ad and round to Fig. 10y, leading to a very strong such reduction in the zonal mean (Fig. 10€). Figure 9 emphasises that this is an actual reduction rather than a normalisation artifact. This suggests that the increase in MF observed during this period is not due to increased dominance of any one species, but instead due to a significant increase in MF per wave carried by all four types, dominating over a reduced absolute number of waves. A possible alternative explanation could be that large-amplitude peaks in the $T^{\prime}\left(k_{z}\right)$ distribution "drown out" smaller peaks which would be visible in other seasons, leading to an apparent reduction in the total number observed; however, the drop appears to apply to all four species, and this effect would therefore have to be extremely large to explain the overall reduction.

\subsection{Differences between generated and observed species}

It would be tempting to conclude that the variation of the species we observe is due primarily to source mechanisms operating at tropospheric and near-surface altitudes. However, while this may be the case in many places and times, we cannot generally assume this.

A particular example of a process which will lead to a wave being generated with one species but being observed as another is illustrated in Fig. 11, adapted from Nappo (2002). Here, we illustrate how critical-layer wind filtering and the consequent refraction of waves just below such a critical layer of gravity waves would lead to a wave observed at a low altitude with type L (i.e. long-vertical-wavelength) would be observed at a height nearer a critical level with type $\mathrm{S}$ (i.e. short-vertical-wavelength).

The wave initially propagates along a given group velocity vector $\boldsymbol{v}_{\mathrm{g} 1}$, with wavefronts as indicated on the diagram. An observation taken here, indicated by the dashed oval, will measure a relatively long vertical wavelength due to the large difference in geometric height between the wavefronts, with some compression of the vertical wavefronts due to the approaching critical level.

At some later time, the wave has propagated some distance and approaches a critical layer $z_{\mathrm{c}}$. As the wave approaches this layer, the vertical component of the group velocity $\boldsymbol{v}_{\mathrm{g} 2}$ tends towards zero, causing the wavefronts to realign. Were the observation (dashed oval) to be taken here instead of at the first location, a much shorter vertical wavelength would be measured.

As a result, the HIRDLS observation will show it to instead be of type $S$ in the second instance and type $L$ in the first, despite having the same source. Furthermore, an observation slightly higher in altitude will not observe the wave at all, consequently reducing the number of observed waves. Analogous processes could also operate in the horizontal direction (not illustrated), or could operate to take a wave packet completely out of our observational filter, causing it

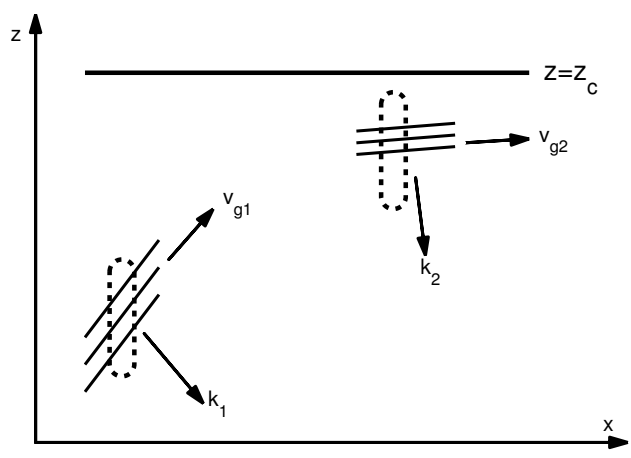

Figure 11. Diagram illustrating an example of a process whereby wind shear would cause the same wave to be observed at two different vertical wavelengths. Adapted from Nappo (2002). See text for details.

to disappear from the results despite remaining present in the physical atmosphere.

Such processes could serve to explain at least some cases where we see a reduction of waves in one type and a compensating increase in waves of another type; in such situations, it may be the case that the generation mechanism has remained constant throughout, but wind layers have altered the wave properties before the point of observation. Many other similar and dissimilar mechanisms may operate, and may operate multiple times on the same wave as it travels through the atmosphere; we only include one example to illustrate this likelihood. While our observed variations in the relative number of each wave may be indicative of source terms, there are many confounding geophysical mechanisms even assuming a perfect instrument.

\section{Discussion and conclusions}

In this study, we have presented an analysis of a full year of HIRDLS data using an overlapping gravity wave detection methodology, including a detailed description of the associated caveats, and explained why our results and methodology differ from previous studies by allowing for the detection of multiple overlapping wavelike signals in a profile. We further identify a series of anomalous features in the observed data, and demonstrate that these most probably arise from the known effects of the HIRDLS launch anomaly, that the signals produced by this anomaly rarely if ever dominate over the largest geophysical signals, and that they contribute at most $10-12 \%$ of the observed signals, all of low amplitude.

We then considered the distribution of the number of observed waves as a function of both $k_{h}$ and $k_{z}$, in the global mean and as a function of altitude and latitude. We showed that the observed waves decreased in vertical wavenumber and increased in horizontal wavenumber with increasing altitude; the latter effect may be consistent with noise effects and should be treated with appropriate caution. Also, significant 
differences are seen in the mean number of observed waves at higher latitudes, arising most probably due to the polarorbiting scan pattern of the instrument and because gravity waves are able to attain longer horizontal wavelengths at low latitudes due to the reduced Coriolis parameter. We note that our results in regard to horizontal wavenumber may be skewed by multiple soundings of long horizontal waves in a proportion of cases, skewing the resulting distribution to longer horizontal waves. We further showed that, once this latitudinal variation was compensated for, significant regional and seasonal variations in the number of observed waves exist.

We then divided these waves into four species, in order to demonstrate the similarities and differences between the temporal and spatial evolution of the wave spectrum. In particular, two of these types, Ls (longvertical-short-horizontal-wavelength) and $\mathrm{Ll}$ (long-verticallong-horizontal-wavelength), were demonstrated to be of especial geophysical importance, the former due to their large momentum flux per packet and the latter to their large temperature perturbations, and consequently potential energy, per wave packet.

Finally, we examined the temporal and spatial variations of these species. In particular, these suggest that the large momentum flux signal of the monsoon appear to be primarily due to variations in the number of Ls waves, whilst the wellstudied Andes hotspot represents an actual reduction relative to the annual mean in the number of observed waves, and consequently a massively increased momentum flux per wave packet. The latter is consistent with previous observations of high wave intermittency in this region (Hertzog et al., 2012; Wright et al., 2013).

Author contributions. C. J. Wright designed and carried out the experiments, and wrote the text. S. M. Osprey provided the initial idea of decomposing the data by wavenumber and useful comments at all stages of data analysis and writing. J. C. Gille provided the data, provided additional data helping to confirm the probable cause of the anomalous peaks, and helped to interpret the results. All authors contributed to proofreading and checking of the final article version.

Acknowledgements. C. J. Wright is currently supported by NERC grant NE/K015117/1. S. M. Osprey is funded by the UK National Centre for Atmospheric Science. C. J. Wright and J. C. Gille were supported by NASA's Aura satellite program under contract NAS59704 for part of this work.

Portions of this study were produced during an extended academic visit by C. J. Wright to the University of Oxford, generously arranged by L. Gray. Several figures use the ColorBrewer colour tables (Harrower and Brewer, 2003). This work would not have been possible without many years of dedicated work by the whole HIRDLS team to correct for the blockage-induced issues with the data.

The National Center for Atmospheric Research is sponsored by the National Science Foundation. Any opinions, findings and conclusions or recommendations expressed in the publication are those of the authors and do not necessarily reflect the views of the National Science Foundation.

Edited by: T. J. Dunkerton

\section{References}

Alexander, M. J.: Interpretations of observed climatological patterns in stratospheric gravity wave variance, J. Geophys. Res. Atmos., 103, 8627-8640, doi:10.1029/97JD03325, 1998.

Alexander, M. J., Gille, J., Cavanaugh, C., Coffey, M., Craig, C., Dean, V., Eden, T., Francis, G., Halvorson, C., Hannigan, J., Khosravi, R., Kinneson, D., Lee, H., Massie, S., Nardi, B., and Lambert, A.: Global Estimates of Gravity Wave Momentum Flux from High Resolution Dynamics Limb Sounder (HIRDLS) Observations, J. Geophys. Res.-Atmos., 113 D15S18, doi:10.1029/2007JD008807, 2008.

Alexander, M. J., Geller, M., McLandress, C., Polavarapu, S., Preusse, P., Sassi, F., Sato, K., Eckermann, S., Ern, M., Hertzog, A., Kawatani, Y., Pulido, M., Shaw, T. A., Sigmond, M., Vincent, R., and Watanabe, S.: Recent developments in gravity-wave effects in climate models and the global distribution of gravitywave momentum flux from observations and models, Q. J. Roy. Meteorol. Soc., 136, 1103-1124, doi:10.1002/qj.637, 2010.

Ern, M. and Preusse, P.: Gravity wave momentum flux spectra observed from satellite in the summertime subtropics: Implications for global modeling, Geophys. Res. Lett., 39, L15810, doi:10.1029/2012GL052659, 2012.

Ern, M., Preusse, P., Alexander, M., and Warner, C. D.: Absolute values of gravity wave momentum flux derived from satellite data, J. Geophys. Res.-Atmos., 109, D20103, doi:10.1029/2004JD004752, 2004.

Ern, M., Preusse, P., Gille, J. C., Hepplewhite, C. L., Mlynczak, M. G., Russell, J. M., and Riese, M.: Implications for atmospheric dynamics derived from global observations of gravity wave momentum flux in stratosphere and mesosphere, J. Geophys. Res.-Atmos., 116, 1-24, doi:10.1029/2011JD015821, 2011.

Fetzer, E. J. and Gille, J. C.: Gravity Wave Variance in LIMS Temperatures. part I: Variability and Comparison with Background Winds, J. Atmos. Sci., 51, 2461-2483, 1994.

France, J., Harvey, V., Alexander, M., Randall, C., and Gille, J.: High Resolution Dynamics Limb Sounder observations of the gravity wave-driven elevated stratopause in 2006, J. Geophys Res.-Atmos., 117, D20108, doi:10.1029/2012JD017958, 2012.

Fritts, D. C.: Gravity wave saturation in the middle atmosphere: A review of theory and observations, Rev. Geophys., 22, 275-308, doi:10.1029/RG022i003p00275, 1984.

Fritts, D. C. and Alexander, M. J.: Gravity Wave Dynamics and Effects in the Middle Atmosphere, Rev. Geophys., 41, 1003, doi:10.1029/2001RG000106, 2003.

Geller, M. A., Alexander, M. J., Love, P. T., Bacmeister, J. T., Ern, M., Hertzog, A., Manzini, E., Preusse, P., Sato, K., Scaife, A. A., and Zhou, T.: A Comparison between Gravity Wave Momentum Fluxes in Observations and Climate Models, J. Climate, 26, 6383-6405, doi:10.1175/JCLI-D-12-00545.1, 2013. 
Gille, J., Barnett, J., Whitney, J., Dials, M., Woodard, D., Rudolf, W., Lambert, A., and Mankin, W.: The High Resolution Dynamics Limb Sounder (HIRDLS) Experiment on Aura, Proc. SPIE, 5152, 162-171, 2003.

Gille, J. C., Barnett, J. J., Arter, P., Barker, M., Bernath, P. F., Boone, C. D., Cavanaugh, C. C., Chow, J., Coffey, M. T., Craft, J., Craig, C., Dials, M., Dean, V., Eden, T. D., Edwards, D. P., Francis, G., Halvorson, C. M., Harvey, V. L., Hepplewhite, C. L., Khosravi, R., Kinnison, D. E., Krinsky, C., Lambert, A., Lee, H., Lyjak, L., Loh, J., Mankin, W., Massie, S. T., McInerney, J., Moorhouse, J., Nardi, B., Packman, D., Randall, C. E., Reburn, W. J., Rudolf, W., Schwartz, M. J., Serafin, J., Stone, K. A., Torpy, B., Walker, K. A., Waterfall, A., Watkins, R. E. J., Whitney, J., Woodard, D., and Young, G.: High Resolution Dynamics Limb Sounder: Experiment overview, recovery, and validation of initial temperature data, J. Geophys. Res.-Atmos., 113, D16S43, doi:10.1029/2007JD008824, 2008.

Gille, J. C., Gray, L. J., Cavanaugh, C. C., Coffey, M. T., Dean, V., Halvorson, C. M., Karol, S., Khosravi, R., Kinnison, D. E., Massie, S. T., Nardi, B., Belmonte Rivas, M., Smith, L., Torpy, B., Waterfall, A., and Wright, C. J.: High Resolution Dynamics Limb Sounder Earth Observing System (EOS): Data Description and Quality Version 7, available at: http://www.eos.ucar.edu/ hirdls/data/ (last access: 28 July 2015), 2013.

Harrower, M. and Brewer, C. A.: ColorBrewer.org: An Online Tool for Selecting Colour Schemes for Maps, Cartogr. J., 40, 27-37, doi:10.1179/000870403235002042, 2003.

Hertzog, A., Alexander, M. J., and Plougonven, R.: On the Intermittency of Gravity Wave Momentum Flux in the Stratosphere, J. Atmos. Sci., 69, 3433-3448, doi:10.1175/JAS-D-12-09.1, 2012.

Hoffmann, L. and Alexander, M. J.: Retrieval of stratospheric temperatures from Atmospheric Infrared Sounder radiance measurements for gravity wave studies, J. Geophys. Res.-Atmos., 114, D07105, doi:10.1029/2008JD011241, 2009.

Holton, J. R., Haynes, P. H., Mcintyre, M. E., Douglass, A. R., Rood, R. B., and Pfister, L.: Stratosphere-Troposphere exchange, Rev. Geophys., 33, 403-439, 1995.

Jiang, J. H., Wang, B., Goya, K., Hocke, K., Eckermann, S. D., Ma, J., Wu, D. L., and Read, W. G.: Geographical distribution and interseasonal variability of tropical deep convection: UARS MLS observations and analyses, J. Geophys. Res.-Atmos., 109, D03111, doi:10.1029/2003JD003756, 2004.

Karoly, D. J., Roof, G. L., and Reeder, M. J.: Gravity wave activity association with tropical convection detected in TOGA COARE sounding data, Geophys. Res. Lett., 23, 261-264, 1996.

Khosravi, R., Lambert, A., Lee, H., Gille, J., Barnett, J., Francis, G., Edwards, D., Halvorson, C., Massie, S., Craig, C., Krinsky, C., McInerney, J., Stone, K., Eden, T., Nardi, B., Hepplewhite, C., Mankin, W., and Coffey, M.: Overview and characterization of retrievals of temperature, pressure, and atmospheric constituents from the High Resolution Dynamics Limb Sounder (HIRDLS) measurements, J. Geophys. Res.-Atmos., 114, D20304, doi:10.1029/2009JD011937, 2009.

Kim, Y.-J., Eckermann, S. D., and Chun, H.-Y.: An overview of the past, present and future of gravity-wave drag parametrization for numerical climate and weather prediction models, Atmos. Ocean, 41, 65-98, doi:10.3137/ao.410105, 2003.

Kinnison, D. E., Gille, J., Barnett, J., Randall, C., Harvey, V. L., Lambert, A., Khosravi, R., Alexander, M. J., Bernath, P. F.,
Boone, C. D., Cavanaugh, C., Coffey, M., Craig, C., Dean, V. C., Eden, T., Ellis, D., Fahey, D. W., Francis, G., Halvorson, C., Hannigan, J., Hartsough, C., Hepplewhite, C., Krinsky, C., Lee, H., Mankin, B., Marcy, T. P., Massie, S., Nardi, B., Packman, D., Popp, P. J., Santee, M. L., Yudin, V., and Walker, K. A.: Global observations of $\mathrm{HNO}_{3}$ from the High Resolution Dynamics Limb Sounder (HIRDLS): First results, J. Geophys. Res.-Atmos., 113 , D16S44, doi:10.1029/2007JD008814, 2008.

Li, J. and Zeng, Q.: A unified monsoon index, Geophys. Res. Lett., 29, 115.1-115.4, doi:10.1029/2001GL013874, 2002.

Massie, S., Gille, J., Khosravi, R., Lee, H., Kinnison, D., Francis, G., Nardi, B., Eden, T., Craig, C., Halvorson, C., Coffey, M., Packman, D., Cavanaugh, C., Craft, J., Dean, V., Ellis, D., Barnett, J., Hepplewhite, C., Lambert, A., Manney, G., Strawa, A., and Legg, M.: High Resolution Dynamics Limb Sounder observations of polar stratospheric clouds and subvisible cirrus, J. Geophys. Res.-Atmos., 112, D24S31, doi:10.1029/2007JD008788, 2007.

McDonald, A. J.: Gravity wave occurrence statistics derived from paired COSMIC/FORMOSAT3 observations, J. Geophys. Res.Atmos., 117, 1-12, doi:10.1029/2011JD016715, 2012.

Mertens, C., Russell, J., Mlynczak, M., She, C., Schmidlin, F., Goldberg, R., Lopezpuertas, M., Wintersteiner, P., Picard, R., and Winick, J.: Kinetic temperature and carbon dioxide from broadband infrared limb emission measurements taken from the TIMED/SABER instrument, Adv. Space Res., 43, 15-27, doi:10.1016/j.asr.2008.04.017, 2009.

Nappo, C. J.: An Introduction to Atmospheric Gravity Waves, International Geophysics Series, Academic Press, Waltham, Massachusetts, USA, 2002.

Nardi, B., Gille, J. C., Barnett, J. J., Randall, C. E., Harvey, V. L., Waterfall, A., Reburn, W. J., Leblanc, T., McGee, T. J., Twigg, L. W., Thompson, A. M., Godin-Beekmann, S., Bernath, P. F., Bojkov, B. R., Boone, C. D., Cavanaugh, C., Coffey, M. T., Craft, J., Craig, C., Dean, V., Eden, T. D., Francis, G., Froidevaux, L., Halvorson, C., Hannigan, J. W., Hepplewhite, C. L., Kinnison, D. E., Khosravi, R., Krinsky, C., Lambert, A., Lee, H., Loh, J., Massie, S. T., McDermid, I. S., Packman, D., Torpy, B., Valverde-Canossa, J., Walker, K. A., Whiteman, D. N., Witte, J. C., and Young, G.: Initial validation of ozone measurements from the High Resolution Dynamics Limb Sounder, J. Geophys. Res.-Atmos., 113, D16S36, doi:10.1029/2007JD008837, 2008.

Preusse, P., Eckermann, S. D., and Offermann, D.: Comparison of Global Distributions of Zonal-Mean Gravity, Geophys. Res. Lett., 27, 3877-3880, 2000.

Preusse, P., Dörnbrack, A., Eckermann, S. D., Riese, M., Schaeler, B., Bacmeister, J. T., Broutman, D., and Grossman, K. U.: Space-based measurements of stratospheric mountain waves by CRISTA 1. Sensitivity, analysis method, and a case study, J. Geophys. Res.-Atmos., 107, 8178, doi:10.1029/2001JD000699, 2002.

Preusse, P., Ern, M., Eckermann, S. D., Warner, C. D., Picard, R. H., Knieling, P., Krebsbach, M., Russell III, J. M., Mlynczak, M. G., Mertens, C. J., and Riese, M.: Tropopause to mesopause gravity waves in August: Measurement and modeling, J. Atmos. Sol.Terr., 68, 1730-1751, doi:10.1016/j.jastp.2005.10.019, 2006.

Preusse, P., Eckermann, S. D., and Ern, M.: Transparency of the atmosphere to short horizontal wavelength gravity waves, J. Geo- 
phys. Res.-Atmos., 113, D24104, doi:10.1029/2007JD009682, 2008.

Remsberg, E. E., Marshall, B. T., Garcia-Comas, M., Krueger, D., Lingenfelser, G. S., Martin-Torres, J., Mlynczak, M. G., Russell, J. M., Smith, A. K., Zhao, Y., Brown, C., Gordley, L. L., LopezGonzalez, M. J., Lopez-Puertas, M., She, C. Y., Taylor, M. J., and Thompson, R. E.: Assessment of the quality of the Version 1.07 temperature-versus-pressure profiles of the middle atmosphere from TIMED/SABER, J. Geophys. Res.-Atmos., 113, D17101, doi:10.1029/2008JD010013, 2008.

Richter, J. H., Sassi, F., and Garcia, R. R.: Toward a Physically Based Gravity Wave Source Parameterization in a General Circulation Model, J. Atmos. Sci., 67, 136-156, doi:10.1175/2009JAS3112.1, 2010.

Sato, K. and Dunkerton, T. J.: Estimates of momentum flux associated with equatorial Kelvin and gravity waves, J. Geophys. Res.Atmos., 102, 26247-26261, doi:10.1029/96JD02514, 1997.

Song, I.-S., Chun, H.-Y., Garcia, R. R., and Boville, B. A.: Momentum Flux Spectrum of Convectively Forced Internal Gravity Waves and Its Application to Gravity Wave Drag Parameterization. Part II: Impacts in a GCM (WACCM), J. Atmos. Sci., 64, 2286-2308, doi:10.1175/JAS3954.1, 2007.

Stockwell, R. G.: S-transform analysis of gravity wave activity from a small scale network of airglow imagers, $\mathrm{PhD}$ thesis, The University of Western Ontario, available at: http://adsabs.harvard. edu/cgi-bin/nph-bib_query?bibcode=1999PhDT........98S （last access: 28 July 2015), 1999.

Stockwell, R. G., Mansinha, L., and Lowe, R. P.: Localization of the Complex Spectrum: The S Transform, IEEE T. Signal Proces., 44, 998-1001, 1996.

Trinh, Q. T., Kalisch, S., Preusse, P., Chun, H.-Y., Eckermann, S. D., Ern, M., and Riese, M.: A comprehensive observational filter for satellite infrared limb sounding of gravity waves, Atmos. Meas. Tech., 8, 1491-1517, doi:10.5194/amt-8-1491-2015, 2015.

Wang, L. and Alexander, M. J.: Gravity wave activity during stratospheric sudden warmings in the 2007-2008 Northern Hemisphere winter, J. Geophys. Res.-Atmos., 114, D18108, doi:10.1029/2009JD011867, 2009.

Wang, L., Fritts, D. C., Williams, B. P., Goldberg, R. A., Schmidlin, F. J., and Blum, U.: Gravity waves in the middle atmosphere during the MaCWAVE winter campaign: evidence of mountain wave critical level encounters, Ann. Geophys., 24, 1209-1226, doi:10.5194/angeo-24-1209-2006, 2006.
Wrasse, C., Fechine, J., Takahashi, H., Denardini, C., Wickert, J., Mlynczak, M., Russell, J., and Barbosa, C.: Temperature comparison between CHAMP radio occultation and TIMED/SABER measurements in the lower stratosphere, Adv. Space Res., 41, 1423-1428, doi:10.1016/j.asr.2007.06.073, 2008.

Wright, C. J.: Detection of stratospheric gravity waves using HIRDLS data, $\mathrm{PhD}$ thesis, University of Oxford, available at: http://ora.ouls.ox.ac.uk/objects/uuid: ef4aa65d-67c1-43ac-90de-1b5bda6c8230 (last access: 29 July 2015), 2010.

Wright, C. J.: A one-year seasonal analysis of martian gravity waves using MCS data, Icarus, 219, 274-282, doi:10.1016/j.icarus.2012.03.004, 2012.

Wright, C. J. and Gille, J. C.: HIRDLS observations of gravity wave momentum fluxes over the monsoon regions, J. Geophys. Res.Atmos., 116, D12103, doi:10.1029/2011JD015725, 2011.

Wright, C. J. and Gille, J. C.: Detecting overlapping gravity waves using the S-Transform, Geophys. Res. Lett., 40, doi:10.1002/grl.50378, 2013.

Wright, C. J., Osprey, S. M., Barnett, J. J., Gray, L. J., and Gille, J. C.: High Resolution Dynamics Limb Sounder Measurements of Gravity Wave Activity in the 2006 Arctic Stratosphere, J. Geophys. Res.-Atmos., 115, D02105, doi:10.1029/2009JD011858, 2010.

Wright, C. J., Rivas, M. B., and Gille, J. C.: Intercomparisons of HIRDLS, COSMIC and SABER for the detection of stratospheric gravity waves, Atmos. Meas. Tech., 4, 1581-1591, doi:10.5194/amt-4-1581-2011, 2011.

Wright, C. J., Osprey, S. M., and Gille, J. C.: Global observations of gravity wave intermittency and its impact on the observed momentum flux morphology, J. Geophys. Res.-Atmos., 118, 1098010993, doi:10.1002/jgrd.50869, 2013.

Wu, D. L., Preusse, P., Eckermann, S. D., Jiang, J. H., de la Torre Juarez, M., Coy, L., and Wang, D. Y.: Remote sounding of atmospheric gravity waves with satellite limb and nadir techniques, Adv. Space Res., 37, 2269-2277, doi:10.1016/j.asr.2005.07.031, 2006.

Yan, X., Arnold, N., and Remedios, J.: Global observations of gravity waves from High Resolution Dynamics Limb Sounder temperature measurements: A yearlong record of temperature amplitude and vertical wavelength, J. Geophys. Res.-Atmos., 115, D10113, doi:10.1029/2008JD011511, 2010. 